\title{
Precessing Jet and Large Dust Grains in the V380 Ori NE Star-forming Region
}

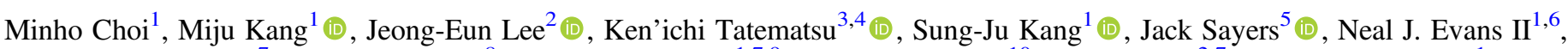 \\ ${\text { Jungyeon } \text { Cho }^{7} \text { (D) , Jungmi Kwon }}^{8}$ (D), Geumsook Park ${ }^{1,7,9}$ (D), Satoshi Ohashi ${ }^{10}$, Hyunju Yoo ${ }^{2,7}$, and Youngung Lee ${ }^{1}$ \\ ${ }^{1}$ Korea Astronomy and Space Science Institute, 776 Daedeokdaero, Yuseong, Daejeon 34055, Korea; minho@kasi.re.kr \\ ${ }^{2}$ School of Space Research, Kyung Hee University, 1732 Deogyeong-daero, Giheung-gu, Yongin-si, Gyeonggi-do 17104, Korea \\ ${ }^{3}$ National Astronomical Observatory of Japan, National Institutes of Natural Sciences, 2-21-1 Osawa, Mitaka, Tokyo 181-8588, Japan \\ ${ }^{4}$ Department of Astronomical Science, SOKENDAI (The Graduate University for Advanced Studies), 2-21-1 Osawa, Mitaka, Tokyo 181-8588, Japan \\ ${ }^{5}$ Department of Physics, Math, and Astronomy, California Institute of Technology, Pasadena, CA 91125, USA \\ ${ }^{6}$ Department of Astronomy, University of Texas at Austin, 2515 Speedway, Stop C1400, Austin, TX 78712-1205, USA \\ ${ }^{7}$ Department of Astronomy and Space Science, Chungnam National University, 99 Daehak-ro, Yuseong-gu, Daejeon, 34134, Korea \\ ${ }^{8}$ Institute of Space and Astronautical Science, Japan Aerospace Exploration Agency, 3-1-1 Yoshinodai, Chuo-ku, Sagamihara, Kanagawa 252-5210, Japan \\ ${ }^{9}$ Department of Physics and Astronomy, Seoul National University, Seoul 151-747, Korea \\ ${ }^{10}$ The Institute of Physical and Chemical Research (RIKEN), 2-1 Hirosawa, Wako-shi, Saitama 351-0198, Japan \\ Received 2017 May 25; revised 2017 September 7; accepted 2017 September 7; published 2017 October 9
}

\begin{abstract}
The V380 Ori NE bipolar outflow was imaged in the $\mathrm{SiO}$ and $\mathrm{CO} J=1 \rightarrow 0$ lines, and dense cores in L1641 were observed in the $2.0-0.89 \mathrm{~mm}$ continuum. The highly collimated $\mathrm{SiO}$ jet shows point-symmetric oscillation patterns in both position and velocity, which suggests that the jet axis is precessing and the driving source may belong to a non-coplanar binary system. By considering the position and velocity variabilities together, accurate jet parameters were derived. The protostellar system is viewed nearly edge-on, and the jet has a flow speed of $\sim 35 \mathrm{~km} \mathrm{~s}{ }^{-1}$ and a precession period of $\sim 1600$ years. The $\mathrm{CO}$ outflow length gives a dynamical timescale of $\sim 6300$ years, and the protostar must be extremely young. The inferred binary separation of 6-70 au implies that this protobinary system may have been formed through the disk instability process. The continuum spectra of L1641 dense cores indicate that the emission comes from dust, and the fits with modified blackbody functions give emissivity power indices of $\beta=0.3-2.2$. The emissivity index shows a positive correlation with the molecular line width, but no strong correlation with bolometric luminosity or temperature. V380 Ori NE has a particularly low value of $\beta=0.3$, which tentatively suggests the presence of millimeter-sized dust grains. Because the dust growth takes millions of years, much longer than the protostellar age, this core may have produced large grains in the starless core stage. HH 34 MMS and HH 147 MMS also have low emissivity indices.
\end{abstract}

Key words: dust, extinction - ISM: individual objects (V380 Ori NE) - ISM: jets and outflows - ISM: structure stars: formation - stars: protostars

\section{Introduction}

L1641 is an active site of star formation, located in the southern part of the Orion A giant molecular cloud. The L1641 region contains thousands of low- and intermediate-mass young stellar objects (YSOs) but no high-mass stars (Strom et al. 1989, 1993; Hsu et al. 2012, 2013). Submillimeter continuum surveys revealed about 60 dense cores in the L1641 cloud, many of them containing protostars (Johnstone \& Bally 2006; Nutter \& Ward-Thompson 2007). Furlan et al. (2016) identified and characterized more than a hundred protostars in L1641. Some of them have unusually red spectral energy distributions (SEDs) and may be extremely young protostars (Stutz et al. 2013).

The ongoing star formation activities in L1641 are manifested by numerous YSO outflows (Morgan et al. 1991; Stanke et al. 2002; Davis et al. 2009). Levreault (1988) found a $\mathrm{CO}$ bipolar outflow located to the northeast of V380 Ori and named it V380 Ori NE, but could not identify the driving source. Later studies showed that this outflow is driven by a deeply embedded protostar (Davis et al. 2000), one of the extreme Class 0 protostars reported by Stutz et al. (2013). This protostar, HOPS 169, has a bolometric luminosity of $L_{\mathrm{bol}}=3.9$ $L_{\odot}$ and bolometric temperature of $T_{\text {bol }}=33 \mathrm{~K}$ (Furlan et al. 2016). Maps of the infrared $\mathrm{H}_{2}$ line and $\mathrm{CO} J=4 \rightarrow 3$ line revealed that the V380 Ori NE outflow is a highly collimated jet flowing along a point-symmetric curve (Davis et al. 2000;
Stanke et al. 2002). Davis et al. (2000) suggested that the outflow variability may be caused by either jet deflection by dense ambient cloud or precession of the driving source. In addition to the very cold SED and highly collimated variable jet, the V380 Ori NE outflow is unusually bright in the $\mathrm{CH}_{3} \mathrm{OH}$ lines (Kang et al. 2013). These characteristics warrant detailed studies of the V380 Ori NE system, which can help to understand the structure and activities of protostars in the earliest stage of star formation.

Gibb et al. (2004) surveyed protostellar outflows in the $\mathrm{SiO}$ $J=5 \rightarrow 4$ line and found that V380 Ori NE shows the SiO emission only at high velocities. The lack of emission from the ambient cloud makes it possible to study the detailed properties of the outflow without ambiguity. These properties are in turn useful for probing the physics of the outflow-launching region, namely the inner part of the accretion disk. Studies of $\mathrm{SiO}$ jets in other star-forming regions have indeed been fruitful in understanding star formation activities, such as the massejection history, shock propagation, jet collimation, jet bending, and jet rotation (Dutrey et al. 1997; Choi 2005; Cabrit et al. 2007; Codella et al. 2007; Choi et al. 2011a; Lee et al. 2015).

In this paper, we present the results of our observations of the V380 Ori NE region with the Very Large Array (VLA) of the National Radio Astronomy Observatory (NRAO), the Taeduk Radio Astronomy Observatory (TRAO), and the 
Caltech Submillimeter Observatory (CSO). We describe the observations in Section 2. In Section 3, we report the results of $\mathrm{SiO}$ imaging with VLA, molecular line spectroscopy and mapping with TRAO, and millimeter-submillimeter continuum imaging with CSO. In Section 4, we discuss the star-forming activities of V380 Ori NE and other nearby objects. A summary is given in Section 5.

\section{Observations}

\subsection{VLA}

The V380 Ori NE region was observed using the VLA in the $\mathrm{SiO} v=0 \quad J=1 \rightarrow 0$ line $(43.423858 \mathrm{GHz})$. Twenty-two antennas were used in the D-array configuration on 2006 January 5 . The spectral window was set to have 32 channels with a channel width of $0.39 \mathrm{MHz}$, giving a velocity resolution of $2.7 \mathrm{~km} \mathrm{~s}^{-1}$.

The nearby quasar 0541-056 (QSO B0539-057) was observed to determine the phase and to obtain the bandpass response. The flux was calibrated by observing the quasar $0319+415$ (3C 84) and setting its flux density to $7.92 \mathrm{Jy}$. A comparison of the amplitude gave a flux density of $1.26 \mathrm{Jy}$ for $0541-056$. To avoid any degradation of the sensitivity owing to pointing errors, the pointing was checked by observing the calibrators at the $\mathrm{X}$ band $(\lambda=3.6 \mathrm{~cm})$. This pointing was performed about once an hour, and just before observing the flux calibrator.

A two-field mosaic technique was used. The phase tracking centers were $\left(\alpha_{2000}, \delta_{2000}\right)=\left(05^{\mathrm{h}} 36^{\mathrm{m}} 36^{\mathrm{s}} .1,-06^{\circ} 38^{\prime} 37^{\prime \prime} \cdot 4\right)$ and $\left(05^{\mathrm{h}} 36^{\mathrm{m}} 36^{\mathrm{s}} \cdot 1,-06^{\circ} 39^{\prime} 08^{\prime \prime} 6\right)$ for the first and the second fields, respectively. The angular distance between the field centers (31"!2) corresponds to the half width of the primary beam. Each field was imaged using a CLEAN algorithm. With a natural weighting, the visibility data of the first and the second fields produced synthesized beams of 2 ." $3 \times 1$ ". 5 and 2 ". $2 \times 1$ ". 5 , respectively, in full width at half-maximum (FWHM). At the end of the CLEAN process, the maps were restored using a circular Gaussian beam of FWHM $=1$ !" 9 for both fields. The maps were corrected for the primary beam response and combined to produce a mosaic map. The intensity of the mosaic map was then tapered toward the edge of the field of view to make the noise level roughly uniform across the map. The imaging was also performed with a robust weighting, using a restoring beam of FWHM $=1$ " 6 . The tapered mosaic maps have rms noise levels of $\sim 1.4 \mathrm{mJy}^{\text {beam }}{ }^{-1}$ for the natural weighting and $\sim 1.5 \mathrm{mJy}^{\text {beam }}{ }^{-1}$ for the robust weighting. There is no continuum emission at a detectable level.

Another protostellar outflow, HH 340/343, was observed in the same observing track, to study the precessing jet driven by IRAS 03256+3055 (Hodapp et al. 2005). The phase-tracking center was $\left(03^{\mathrm{h}} 28^{\mathrm{m}} 45^{\mathrm{s}} 3,31^{\circ} 05^{\prime} 42^{\prime \prime} 0\right)$, and the phase calibrator was $0336+323$ (PKS $0333+321$ ). The bootstrapped flux density of $0336+323$ was $0.79 \mathrm{Jy}$. The image noise level of the HH 340/343 region is similar to that of the V380 Ori NE region. The $\mathrm{SiO}$ line was not detected. We omit further description of the $\mathrm{HH} 340 / 343$ region in this paper.

\subsection{TRAO}

The V380 Ori NE region was observed using the Second Quabbin Optical Imaging Array (SEQUOIA-TRAO; Erickson et al. 1999) receiver system on the TRAO $14 \mathrm{~m}$ telescope. The data were obtained in 2016 January-February, during an initial
Table 1

TRAO Telescope Parameters

\begin{tabular}{lcc}
\hline \hline $\begin{array}{l}\text { Frequency } \\
(\mathrm{GHz})\end{array}$ & $\begin{array}{c}\text { Beam FWHM } \\
(\operatorname{arcsec})\end{array}$ & $\begin{array}{c}\text { Efficiency } \\
\eta_{\mathrm{mb}}\end{array}$ \\
\hline 86.24 & 60 & 0.46 \\
98.00 & 53 & 0.52 \\
115.27 & 45 & 0.51 \\
\hline Note. & \\
${ }^{a}$ Frequency where the beam size and main-beam efficiency were measured.
\end{tabular}

test period after the receiver array was relocated from the Five College Radio Astronomy Observatory. The backend consisted of an FFT spectrometer. The spectral window has 4096 channels in a $62.5 \mathrm{MHz}$ bandwidth. The telescope parameters are listed in Table 1. Telescope pointing was checked by observing Orion IRc2 (Baudry et al. 1995) in the $\mathrm{SiO} v=1$ $J=2 \rightarrow 1$ maser line. The pointing observations were performed about once an hour. The typical pointing error was $\sim 8^{\prime \prime}$. The antenna temperature was calibrated by the standard chopper-wheel method, which automatically corrected for the effects of atmospheric attenuation. We will present the TRAO data in the main-beam temperature $\left(T_{\mathrm{mb}}\right)$ scale.

The central source was observed in the $\mathrm{H}^{13} \mathrm{CO}^{+} J=1 \rightarrow 0$ line $(86.754294 \mathrm{GHz})$ and the $\mathrm{C}^{34} \mathrm{~S} \quad J=2 \rightarrow 1$ line $(96.412982 \mathrm{GHz})$, using the reference pixel of the receiver array. The spectral channel width corresponds to a velocity width of 0.053 and $0.047 \mathrm{~km} \mathrm{~s}^{-1}$, respectively. Typical system temperatures were 200 and $180 \mathrm{~K}$, respectively. The target position was $\left(\alpha_{2000}, \delta_{2000}\right)=\left(05^{\mathrm{h}} 36^{\mathrm{m}} 36^{\mathrm{s}} .1, \quad-06^{\circ} 38^{\prime} 53^{\prime \prime}\right)$, which corresponds to the submillimeter continuum source (Davis et al. 2000). The data were taken by position switching with the reference position of $(\Delta \alpha, \Delta \delta)=\left(-40^{\prime},-40^{\prime}\right)$ relative to the target position. For each spectrum, a first-order baseline was removed. The spectral baseline was determined from the velocity intervals of $(3,5)$ and $(9,11) \mathrm{km} \mathrm{s}^{-1}$.

The $10^{\prime} \times 10^{\prime}$ region around the target position was mapped in the $\mathrm{CO} J=1 \rightarrow 0$ line $(115.271204 \mathrm{GHz})$. The typical system temperature was $\sim 580 \mathrm{~K}$. The data were taken by onthe-fly mapping (see Jackson et al. 2006 for details of the mapping mode) with the reference position of $(\Delta \alpha$, $\Delta \delta)=\left(-90^{\prime},-90^{\prime}\right)$. Scans along the right ascension and declination were alternated. The data were regridded onto a $25^{\prime \prime}$ grid covering the mapping region. For each spectrum, a firstorder baseline was removed. The spectral baseline was determined from the velocity intervals of $(-21,-7)$ and (21, $35) \mathrm{km} \mathrm{s}^{-1}$. One of the aims of the CO mapping was to check the data reliability. The resulting $\mathrm{CO}$ map and spectra are consistent with those presented by Morgan et al. (1991).

\section{3. $\mathrm{CSO}$}

The northern part of the L1641 cloud was observed using the CSO $10.4 \mathrm{~m}$ telescope in 2014 November-December. The data were obtained using the Multiwavelength Submillimeter Inductance Camera (MUSIC; Golwala et al. 2012; Sayers et al. 2014, 2016) during a commissioning run of the instrument. Detailed observational parameters are listed in Table 2.

The data were taken by scanning the sky in a raster pattern. Scans along the right ascension and declination were alternated. Four wavelength bands were observed simultaneously. The 
Table 2

Summary of the MUSIC Parameters

\begin{tabular}{lccccc}
\hline \hline Band & $\begin{array}{c}\text { Wavelength } \\
(\mathrm{mm})\end{array}$ & $\begin{array}{c}\text { Beam } \\
\text { FWHM }\end{array}$ & $\epsilon^{\mathrm{a}}$ & $\begin{array}{c}\eta_{\mathrm{hpf}}^{\mathrm{b}} \\
\left(\mathrm{Jy} \mathrm{beam}^{-1}\right)\end{array}$ \\
\hline 0 & 2.0 & $48^{\prime \prime}$ & $6.0 \%$ & 0.89 & 0.10 \\
1 & 1.4 & $36^{\prime \prime}$ & $4.6 \%$ & 0.92 & 0.11 \\
2 & 1.1 & $32^{\prime \prime}$ & $5.5 \%$ & 0.93 & 0.2 \\
3 & 0.89 & $29^{\prime \prime}$ & $9.4 \%$ & 0.94 & 1.0 \\
\hline
\end{tabular}

Notes. See Sayers et al. (2016) for more details.

${ }^{a}$ Uncertainty in the absolute flux calibration.

${ }^{b}$ Correction factor for the high-pass filter, i.e., the ratio of the peak source amplitude in the filtered map compared with the nominal peak source amplitude.

${ }^{\mathrm{c}}$ Noise rms level in each map.

target mapping region was a $60^{\prime} \times 60^{\prime}$ square centered at $\left(\alpha_{2000}, \quad \delta_{2000}\right)=\left(05^{\mathrm{h}} 36^{\mathrm{m}} 25^{\mathrm{s}} .4, \quad-06^{\circ} 42^{\prime} 59^{\prime \prime}\right)$. Uranus was observed once each night for flux and pointing calibrations. The pointing accuracy was $\sim 5^{\prime \prime}$.

The calibration and imaging were performed by the MUSIC commissioning team (Sayers et al. 2016). The data were processed with a high-pass filter to reduce low-frequency noise, which removes signals contained in Fourier modes larger than $\sim 16^{\prime}$. For the sources in our maps, which are generally smaller than $40^{\prime \prime}$, this effect of the high-pass filter is minimal and can be approximated by the correction factor $\eta_{\mathrm{hpf}}$ listed in Table 2 . (See Section 3.3 for the effect of the filtering on extended sources.) The map of each wavelength band was divided by $\eta_{\text {hpf }}$ to correct for the effect of the high-pass filter.

The correction factors were determined by creating mock images of a point-like source, matching the point-spread function in each band. These images were reverse-mapped into time-ordered data using a scan pattern matching the real observations. The mock data were processed identically to the real time-ordered data, and maps were produced. The peak heights of the source in these maps were compared to those in the original mock images to obtain the $\eta_{\text {hpf }}$ values.

\section{Results}

\subsection{VLA SiO Imaging}

The SiO line emission map (Figure 1) shows the structure of the V380 Ori NE outflow system in the field of view. The highly collimated bipolar jet flows along a gentle curve that clearly shows a point-symmetric morphology. The overall shape of the $\mathrm{SiO}$ jet is consistent with that of the $\mathrm{H}_{2}$ jet (Davis et al. 2000) and the $4.5 \mu \mathrm{m}$ emission features (Figure 1). The velocity channel maps (Figure 2) show that the northern jet is entirely blueshifted and the southern jet is entirely redshifted. This clear separation between the blueshifted and redshifted gas suggests that the outflow collimation angle is smaller than the inclination angle of the outflow axis with respect to the plane of the sky.

At a smaller scale, the $\mathrm{SiO}$ jet consists of a series of emission peaks (Figure 3). The northern jet shows a relatively even intensity distribution. That is, the strongest three peaks (b3, b4, and b6) have comparable intensities. By contrast, in the southern jet, the strongest peak (r6) dominates the intensity distribution. The intensity distribution of the $\mathrm{H}_{2}$ jet shows a similar asymmetry (Davis et al. 2000). The northern $\mathrm{H}_{2}$ jet shows a relatively continuous flow, while the southern $\mathrm{H}_{2}$ jet can be seen only around the brightest knots. The $\mathrm{SiO}$ map shows the outflow structure near the driving source much better than the $\mathrm{H}_{2}$ image, probably because the $\mathrm{H}_{2}$ line is subject to extinction. The spatial distribution of the $\mathrm{SiO}$ outflow peaks shows an undulation pattern. The position angle of the outflow peaks with respect to the driving source seems to oscillate periodically. This pattern is as expected for a precessing outflow. Each of the northern/southern jets in the $\mathrm{SiO}$ image covers more than a full cycle of the oscillation.

To describe the directional variability of the $\mathrm{SiO}$ jet and analyze the oscillation of the position angle, it is necessary to accurately determine the position of the driving source. Among the emission sources known so far, the best indicator for the position of the central protostar is the submillimeter continuum source (Davis et al. 2000), but the angular resolution $\left(\sim 14^{\prime \prime}\right)$ is much worse than that of the $\mathrm{SiO}$ image. The near-infrared position of HOPS 169 given by Furlan et al. (2016) corresponds to an emission knot of the northern outflow (b1), not the protostar itself. Examinations of the near-infrared image (Figure 4) show that there is a local intensity minimum near the midpoint between the outflow peaks b1 and $\mathrm{r} 1$. This feature is elongated in the east-west direction. It seems to be a dark lane caused by the edge-on disk or flattened envelope structure around the protostar. The intersection between the infrared dark lane and the $\mathrm{SiO}$ outflow axis (b1-r1 line) is a good candidate for the protostellar position. The coordinate of this position is $\left(05^{\mathrm{h}} 36^{\mathrm{m}} 36^{\mathrm{s}} .12,-06^{\circ} 38^{\prime} 54^{\prime \prime} \cdot 9\right)$.

Figure 5 shows the $\mathrm{SiO}$ spectra at the outflow peaks. Centroid velocities were derived by fitting each spectrum with a single Gaussian profile. Along each of the northern/southern outflows, the centroid velocity shows an undulation pattern. The spatial scale (wavelength) of the velocity undulation is similar to that of the position angle (Figure 6). The centroid velocity distribution also shows an obvious point symmetry against the driving source. The undulation patterns and point symmetries of the position angle and centroid velocity suggest that the directional variability and the velocity variability are closely related phenomena, most likely having a single root cause. The precession of the jet axis may be a natural explanation. Here we use the term "precessing jet" to describe the oscillating and point-symmetric variability of the jet, which does not necessarily mean that it is caused by the precession of the disk (Section 4.3).

\subsection{TRAO Molecular Line Spectroscopy and Mapping}

The $\mathrm{H}^{13} \mathrm{CO}^{+}$and $\mathrm{C}^{34} \mathrm{~S}$ lines were observed to determine the systemic velocity of the dense cloud core containing the V380 Ori NE protostar. These lines are expected to be optically thin. Figure 7 shows the spectra. Both lines were detected clearly. The $\mathrm{H}^{13} \mathrm{CO}^{+}$line especially shows a well-defined single peak with a good signal-to-noise ratio. The best-fit Gaussian profiles give centroid velocities of $7.05 \pm 0.03 \mathrm{~km} \mathrm{~s}^{-1}$ for $\mathrm{H}^{13} \mathrm{CO}^{+}$and $7.16 \pm 0.06 \mathrm{~km} \mathrm{~s}^{-1}$ for $\mathrm{C}^{34} \mathrm{~S}$. The line widths are $\mathrm{FWHM}=0.55$ and $0.78 \mathrm{~km} \mathrm{~s}^{-1}$, respectively. These velocities are consistent with that of the $C^{18} \mathrm{O} J=3 \rightarrow 2$ line (Wilson et al. 1999). Therefore, the systemic velocity of the V380 Ori NE dense core is determined to be $V_{0}=7.1 \mathrm{~km} \mathrm{~s}^{-1}$.

The CO line was observed to measure the total extent of the V380 Ori NE bipolar outflow. Figure 8 shows the maps of line wings. These maps have a denser sampling and cover a larger area than those presented by Morgan et al. (1991). The length of each outflow lobe is $\sim 140^{\prime \prime}$. Considering the beam size, the 


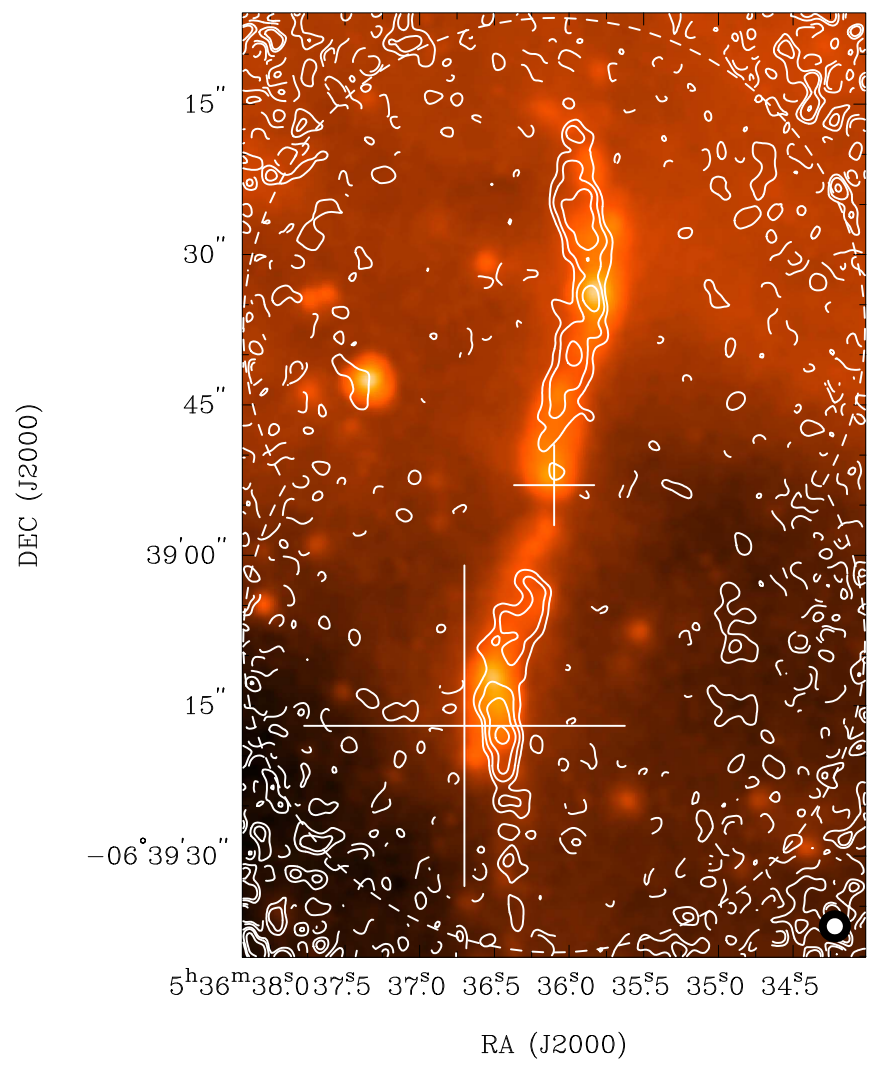

Figure 1. Map of the $\mathrm{SiO} v=0 J=1 \rightarrow 0$ line toward V380 Ori NE, made with a robust weighting and averaged over the velocity range of $V_{\mathrm{LSR}}=(-9.0$, 23.3) $\mathrm{km} \mathrm{s}^{-1}$. Contour levels are $1,2,4$, and $8 \times 1.2 \mathrm{mJy} \mathrm{beam}^{-1}$, and the rms noise is $0.4 \mathrm{mJy}_{\text {beam }}{ }^{-1}$ near the map center. Dashed contours are for negative levels. The intensity taper was not applied, and the noise level is higher at the edge of the map than at the center. Shown in the bottom right-hand corner is the restoring beam: FWHM $=1$ ". 6 . The background heat-scale image shows the $4.5 \mu \mathrm{m}$ band map from Spitzer Space Telescope. Small plus sign: the submillimeter continuum peak (Davis et al. 2000). Large plus sign: the $\mathrm{CH}_{3} \mathrm{OH}$ emission peak KLC 8 (Kang et al. 2013). Dashed arcs: primary beam at each mosaic field.

deconvolved length of the outflow may be $\sim 120^{\prime \prime}$. There is no sign of outflow beyond this length. The $\mathrm{CO}$ emission component in the southwestern corner of Figure 8 corresponds to the cloud associated with NGC 1999. As in the case of the $\mathrm{SiO}$ outflow, the $\mathrm{CO}$ outflow also shows a clear separation between the blueshifted and redshifted gas, which suggests that the $\mathrm{CO}$ outflow must be well-collimated.

\subsection{CSO Continuum Imaging}

Initial inspections of the resulting MUSIC images showed that several emission sources are detectable. Comparison of the source positions in the MUSIC maps with those of submillimeter counterparts (Nutter \& Ward-Thompson 2007) revealed that the position difference is $\sim 4^{\prime \prime}$ on average, which is consistent with the pointing accuracy.

The MUSIC maps match the sensitivity of similar maps in the literature (Johnstone \& Bally 2006; Nutter \& WardThompson 2007; Davis et al. 2009), but provide data in multiple bands, enabling the study of spectral properties. We searched for sources detectable in at least two bands. For a source with a typical spectral slope of dust continuum emission, the signal-to-noise ratio is higher in the $\lambda=1.4$ and $1.1 \mathrm{~mm}$ bands than in the other bands. Figure 9 shows the combined map of the 1.4 and $1.1 \mathrm{~mm}$ bands. The noise levels are not entirely uniform over the mapping region, and there are some patches of relatively high noise. Eight sources were identified, and their properties are listed in Table 3. There are probable sources near the northern edge of the map, but they are ignored because the noise level is high around them. Some of the detected objects, such as L1641N MMS, contain several protostars and may be considered as clumps, while others, such as V380 Ori NE, contain a single protostar (or a protobinary) and may be considered as dense cloud cores. In this paper, we refer to the detected objects as "cores" for simplicity.

There is a cluster of three cores in the $\mathrm{L} 1641 \mathrm{~N}$ region, as shown in Figure 10. One of them, TUKH 74, is a starless core. HH 34 MMS and V380 Ori NE are relatively isolated cores (Figures 11-12). There is another cluster of three cores in the HH 1-2 region (Figure 13). The star formation activities of individual cores are summarized in Appendix. Integrated flux densities were measured for each core. The flux densities and statistical uncertainties are listed in Table 4. The uncertainties are dominated by the statistical uncertainties rather than the calibration uncertainties.

The correction factors for the high-pass filter listed in Table 2 are not exactly correct for extended sources. The strongest and most extended source in the field of view is L1641N MMS. Elliptical Gaussian fits to the 1.4 and $1.1 \mathrm{~mm}$ maps give a deconvolved size of FWHM $\approx 44^{\prime \prime} \times 20^{\prime \prime}$. The correction factors appropriate for this source size are smaller by $\sim 3 \%$. As shown in Table 5, the effect of source size on the flux density measurements is smaller than the uncertainties. Moreover, the amount of this effect is similar across all the wavelength bands, and the spectral slope does not change significantly. The other sources in the field are weaker and smaller than L1641N MMS. Therefore, at the sensitivity level of the MUSIC data, the correction for source size is unnecessary.

\section{Discussion \\ 4.1. V380 Ori NE Precessing Outflow}

The thermal $\mathrm{SiO}$ lines are excellent tracers of the primary jets driven by protostars because the $\mathrm{SiO}$ abundance in the jet is enhanced by several orders of magnitude compared to that of the ambient molecular cloud (Dutrey et al. 1997; Choi 2005; Hirano et al. 2006; Cabrit et al. 2007; Codella et al. 2007; Choi et al. 2011b). The SiO spectra of the V380 Ori NE outflow (Figure 5; also see Figure 6 of Gibb et al. 2004) show that there is no emission component at the systemic velocity, which suggests that the $\mathrm{SiO}$ line exclusively traces the jet. Interferometric images of the $\mathrm{SiO}$ lines often provide valuable information on the spatial and velocity structure of the primary jet. However, the $\mathrm{SiO}$ lines are poor tracers of the mass and related quantities because the abundance varies greatly (Gibb et al. 2004; López-Sepulcre et al. 2016). In this paper, we concentrate on analysis of the morphology and kinematics of the $\mathrm{SiO}$ jet.

To explain the curved morphology of the $\mathrm{H}_{2}$ jet, Davis et al. (2000) suggested that both northern and southern jets are deflected by dense ambient gas at roughly the same distance from the protostar. While the $\mathrm{H}_{2}$ image shows only the brightest part of the jet, the $\mathrm{SiO}$ image shows a more comprehensive picture and seems to rule out the deflection model. A good example of a deflected jet is the asymmetric bipolar jet of NGC 1333 IRAS 4A (Choi 2005). The northeastern jet of IRAS 4A 


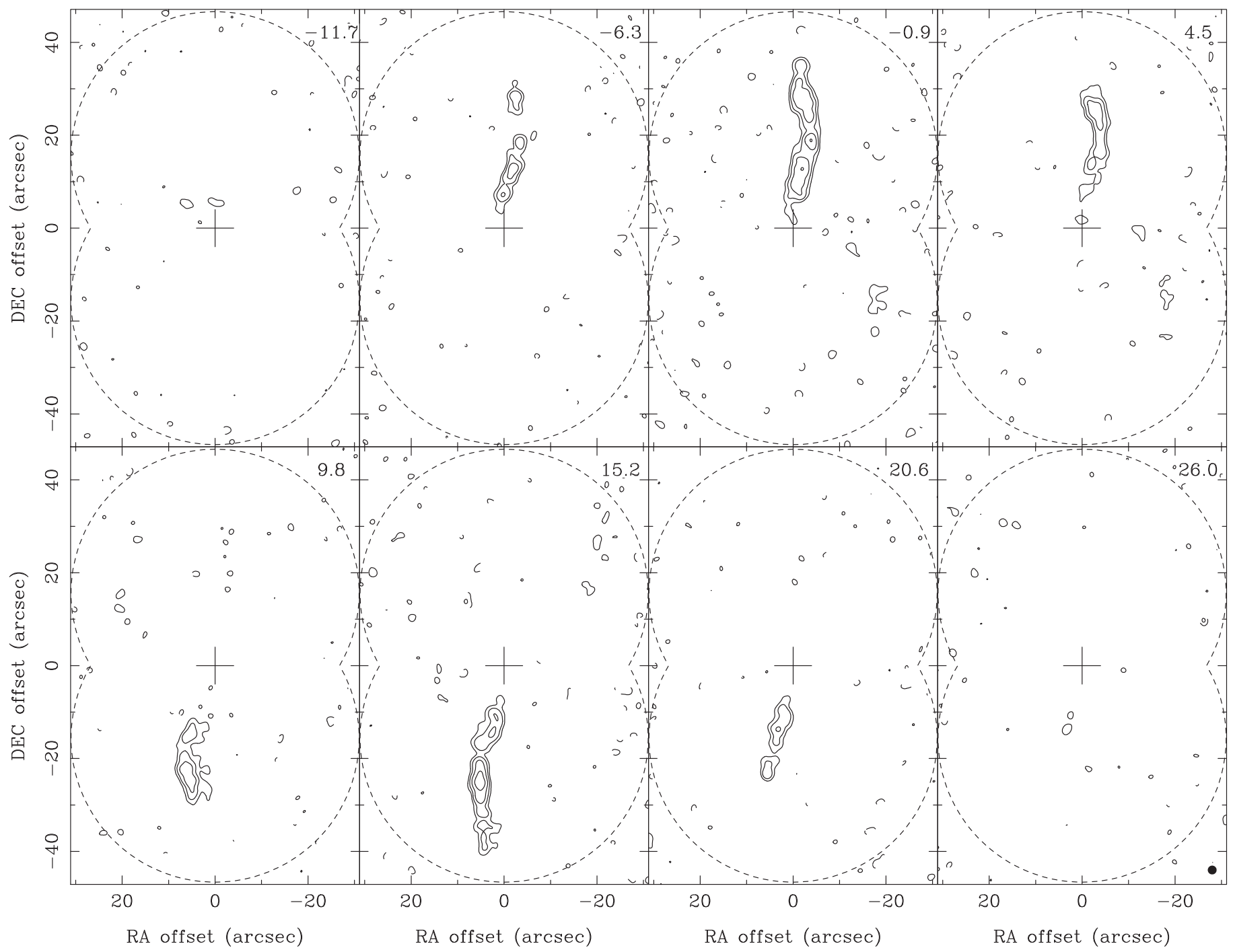

Figure 2. Velocity channel maps of the $\mathrm{SiO}$ line toward V380 Ori NE, made with a natural weighting. Central velocity of each map is written in the top right-hand corner, and the velocity width for each map is $5.4 \mathrm{~km} \mathrm{~s}^{-1}$. Contour levels are $1,2,4$, and $8 \times 3 \mathrm{mJy}_{\mathrm{beam}}^{-1}$, and the rms noise is $1.0 \mathrm{mJy}$ beam ${ }^{-1}$. The intensity is tapered toward the edge of the field of view. Shown in the bottom right-hand corner is the restoring beam: FWHM $=1{ }^{\prime \prime} 9$. Plus sign: the submillimeter continuum peak (Davis et al. 2000).

shows a sharp bend, but the southwestern jet does not. In the case of V380 Ori NE, the point-symmetric patterns of the jet morphology and kinematics suggest that the outflow variability is intrinsic to the driving source. Moreover, the $\mathrm{SiO}$ jet direction and velocity oscillate along smooth curves without any abrupt change, which also supports the intrinsic variability and rules out external perturbation. Among the intrinsic variability mechanisms proposed (Eislöffel \& Mundt 1997; Fendt \& Zinnecker 1998), the precession of a jet axis seems to be the only viable one. The orbital motion of a jet source in a binary system can produce an oscillation pattern, but the jet morphology would show a mirror symmetry (see Figure 2 of Fendt \& Zinnecker 1998). The Lorentz forces caused by external magnetic fields can produce a point-symmetric bending, but the jet would not show oscillations.

To measure quantities describing the variability, the position angle and velocity of the outflow peaks are plotted as functions of the angular distance from the driving source (Figure 6), and they were fitted with sinusoidal functions. The number of free parameters can be reduced by assuming a perfect point symmetry. That is, the northern and southern jets were assumed to have the same oscillation period and amplitudes. The position angle and line-of-sight velocity depend on the inclination angle of the velocity vector at each flow element in a way that their oscillation patterns have a phase difference of $\pi / 2$. The fitting functions are

$$
\begin{gathered}
p=A_{p} \cos \left(\frac{2 \pi d}{L}+\varphi_{0}\right)+D_{p} \\
v_{p}=A_{v} \sin \left(\frac{2 \pi d}{L}+\varphi_{0}\right)+D_{v},
\end{gathered}
$$

where $p$ is the position angle (minus $180^{\circ}$ for the southern jet) with respect to the driving source, $v_{p}$ is the centroid velocity $\left(V_{c}\right)$ with respect to the systemic velocity $\left(V_{c}-V_{0}\right.$ for the southern jet and $V_{0}-V_{c}$ for the northern jet), $A_{p}$ and $A_{v}$ are the oscillation amplitudes, $d$ is the angular distance from the driving source, $L$ is the wavelength of oscillation, $\varphi_{0}$ is the phase of oscillation at the center, and $D_{p}$ and $D_{v}$ are the mean values. Fitting all the data points together, the best-fit values are $L=28^{\prime \prime} \pm 2^{\prime \prime}$, 


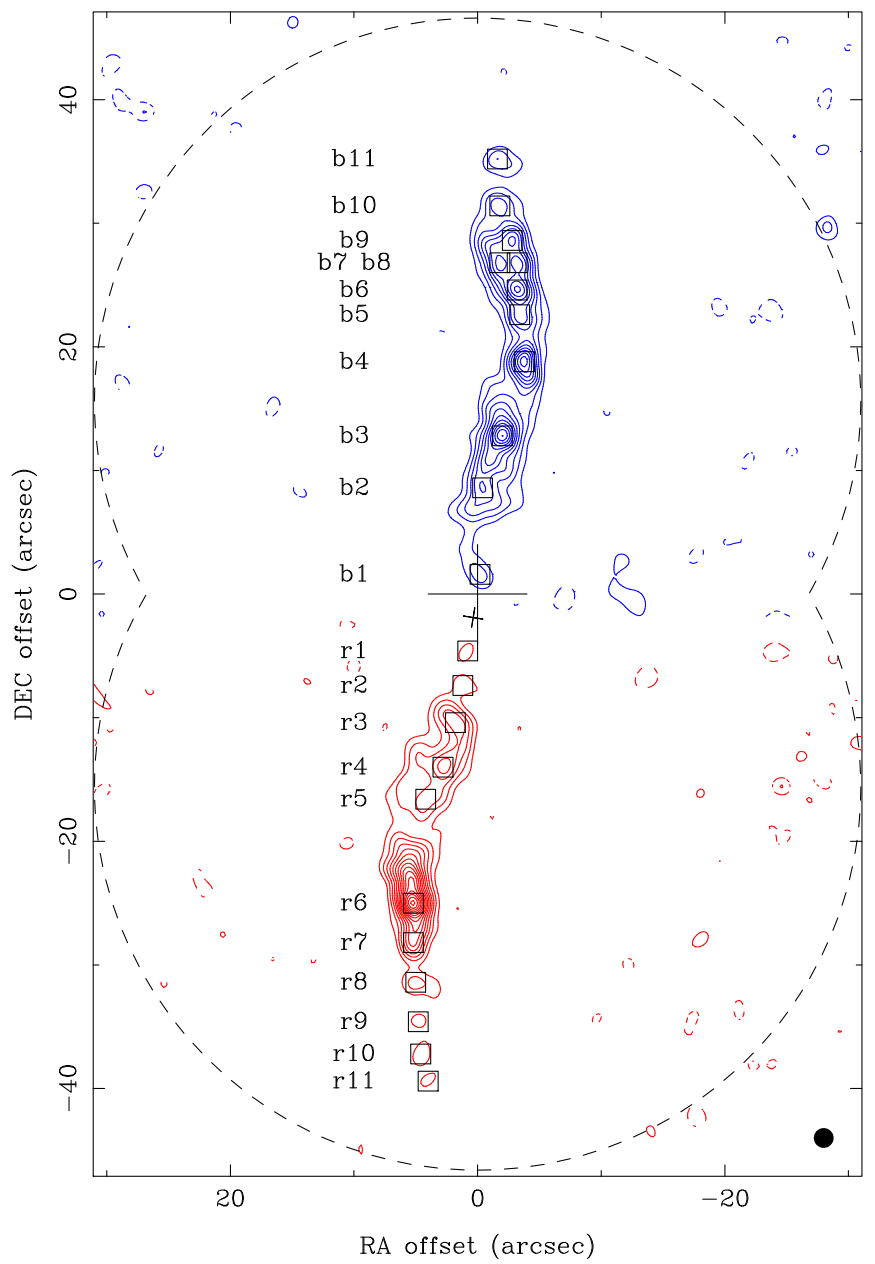

Figure 3. Maps of the $\mathrm{SiO}$ line toward V380 Ori NE, made with a robust weighting. In the northern half of the map, the blue contours show the $\mathrm{SiO}$ line intensity averaged over the velocity range of $(-9.0,7.2) \mathrm{km} \mathrm{s}^{-1}$. In the southern half of the map, the red contours show the intensity averaged over $(7.2,23.3) \mathrm{km} \mathrm{s}^{-1}$. The lowest contour level is $1.8 \mathrm{mJy} \mathrm{beam}^{-1}$, and the contour interval is $1.2 \mathrm{mJy}$ beam $^{-1}$. The intensity is tapered toward the edge of the field of view. Shown in the bottom right-hand corner is the restoring beam: $\mathrm{FWHM}=1$ " 6 . Plus sign: the submillimeter continuum peak (Davis et al. 2000). Small cross: expected position of the protostar (Figure 4). Squares: peak positions of the $\mathrm{SiO}$ emission.

$\varphi_{0}=5.0 \pm 0.6, \quad A_{p}=3.5 \pm 0.5, \quad D_{p}=-8.5 \pm 0.5, \quad A_{v}=$ $2.0 \pm 0.5 \mathrm{~km} \mathrm{~s}^{-1}$, and $D_{v}=8.2 \pm 0.3 \mathrm{~km} \mathrm{~s}^{-1}$.

The first two emission peaks (b1 and r1) have relatively large uncertainties, and there is a large velocity jump between peaks $\mathrm{r} 1$ and $\mathrm{r} 2$. The jump probably indicates that the jet may be still accelerating in this area. To understand whether these first peaks affect the determination of the oscillation parameters, the fit was performed again without these data points. The best-fit values are then $L=28^{\prime \prime}, \varphi_{0}=5.1, A_{p}=3.5, D_{p}=-8^{\circ} .6$, $A_{v}=2.0 \mathrm{~km} \mathrm{~s}^{-1}$, and $D_{v}=8.3 \mathrm{~km} \mathrm{~s}^{-1}$. The differences from the values in the previous paragraph are smaller than the corresponding uncertainties. Therefore, the derived oscillation parameters are robust.

The oscillation parameters can be related to the parameters describing the precessing jet. Assuming that the $\mathrm{SiO}$ outflow peaks are confined to the surface of a circular cone,

$$
\tan A_{p}=\tan \theta_{p} / \cos i_{p}
$$

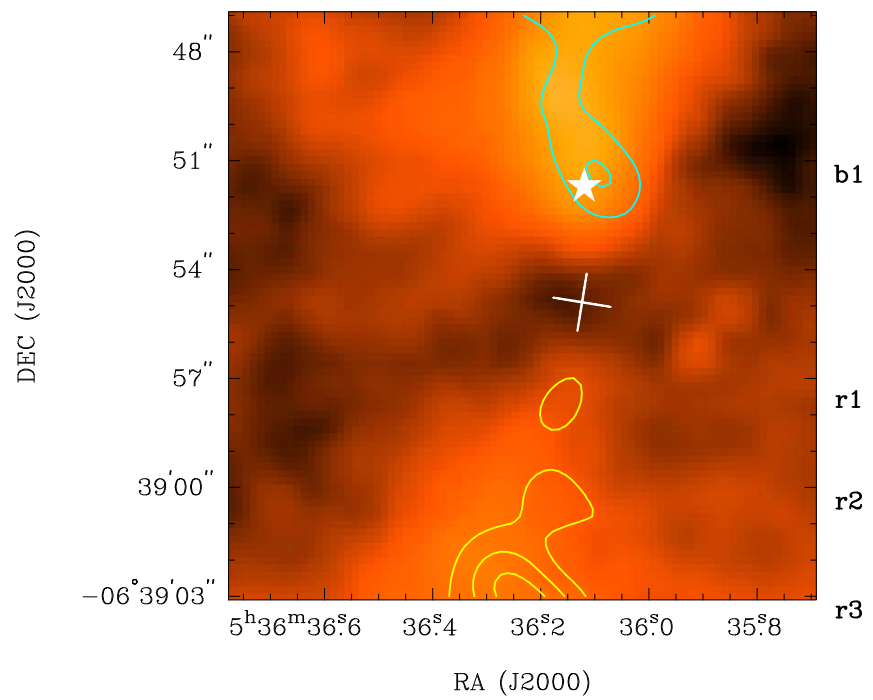

Figure 4. Maps of the $\mathrm{SiO}$ line, as shown in Figure 3, in the central $16^{\prime \prime} \times 16^{\prime \prime}$ region. Contour levels are 1,2 , and $3 \times 1.8 \mathrm{mJy}^{\text {beam }}{ }^{-1}$. The $\mathrm{SiO}$ outflow peaks are labeled on the right edge of the figure. The background heat-scale image shows the $3.6 \mu \mathrm{m}$ band map from Spitzer Space Telescope, with the extended nebulosity subtracted out. Cross: position of the protostar inferred from the outflow axis and infrared dark lane. Star symbol: HOPS 169 (Furlan et al. 2016).

where $\theta_{p}$ is the half-angle of the precession cone opening, and $i_{p}$ is the inclination angle of the precession cone axis with respect to the plane of the sky. Assuming that each flow element is moving ballistically at a uniform speed and along a straight line away from the driving source,

$$
\begin{aligned}
& A_{v}=v_{f} \cos i_{p} \sin \theta_{p} \\
& D_{v}=v_{f} \sin i_{p} \cos \theta_{p},
\end{aligned}
$$

where $v_{f}$ is the flow speed. Solving these equations, $i_{p}=13^{\circ} .5 \pm 3.1, \theta_{p}=3.4 \pm 0.5$, and $v_{f}=35 \pm 8 \mathrm{~km} \mathrm{~s}^{-1}$. The precession cone is close to the plane of the sky, the jetdriving disk may be viewed nearly edge-on, and the velocity vectors of flow elements have inclinations ranging from $10^{\circ}$ to $17^{\circ}$.

The coverage of the $\mathrm{SiO}$ jet in this paper corresponds to $\sim 1.3$ precession cycles. To call the variability strictly "periodic," a significantly longer coverage is necessary. The large-scale structure of the precessing jet (Section 4.2) may be more complicated than the simple description given here, but the jet should be covered over two cycles or more to uncover more details. Future observations over a larger region, by way of either deeper integration in the $\mathrm{SiO}$ line or imaging with a different tracer such as $\mathrm{CH}_{3} \mathrm{OH}$, may be helpful.

Figure 6(a) shows that there is a small but noticeable deviation from the perfect point symmetry. The data points of northern jet mostly lies above those of southern jet. The difference is $\sim 4^{\circ}$. Because this deviation is clearer at larger distances, the position uncertainty of the protostar is not the cause. One possible explanation is a westward motion of the protostar relative to the ambient cloud.

\subsection{Timescales and Evolutionary Status of V380 Ori NE}

The parameters of the precessing jet provide interesting information on the evolutionary status of the V380 Ori NE 


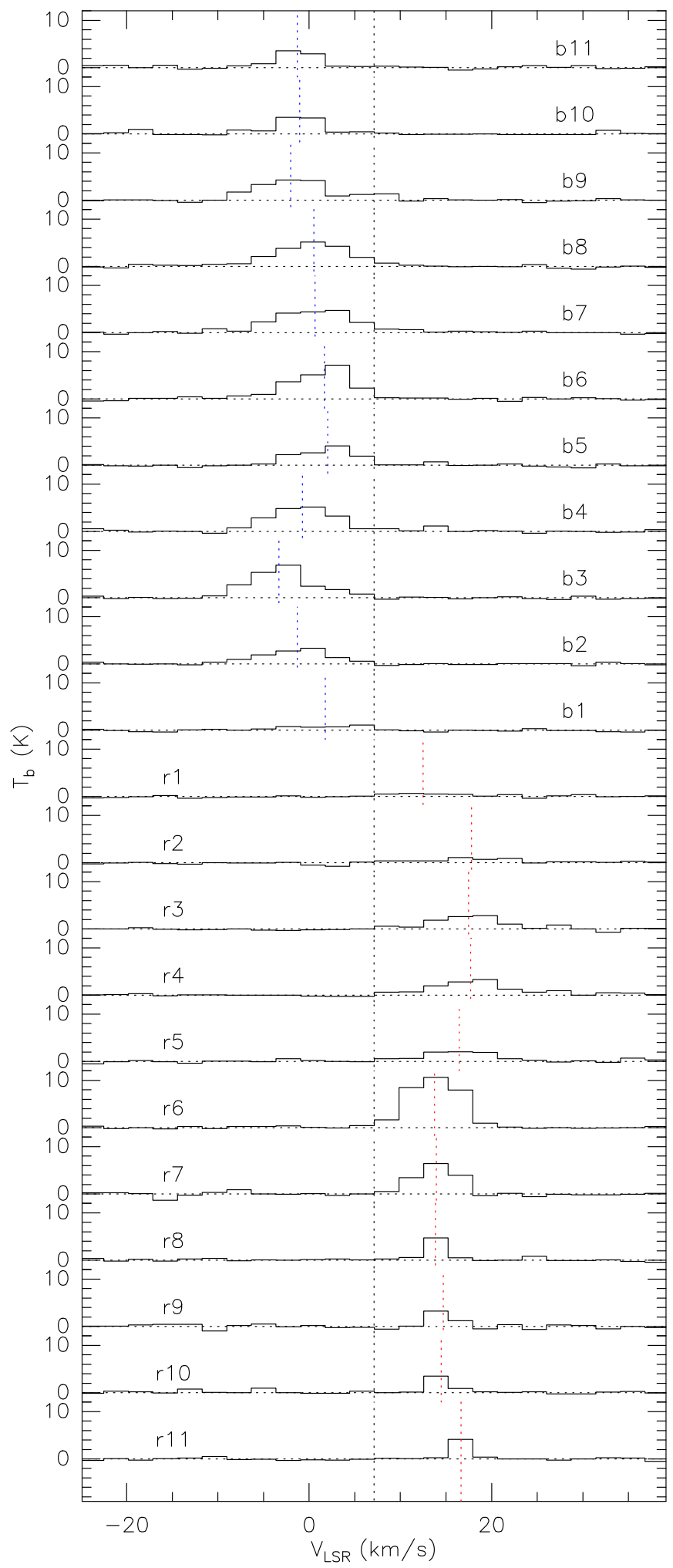

Figure 5. Spectra of the $\mathrm{SiO}$ line at the outflow peaks (see Figure 3 for the positions of the outflow peaks). The spectra were taken from the map made with a natural weighting. The intensity taper was not applied. Vertical black dotted line: systemic velocity of V380 Ori NE, $V_{\mathrm{LSR}}=7.1 \mathrm{~km} \mathrm{~s}^{-1}$ (Section 3.2). Blue/red dotted lines: centroid velocity.

system. Assuming that the distance to V380 Ori NE is the same as that of Orion KL (418 \pm 6 pc; Kim et al. 2008), the oscillation wavelength corresponds to $1.2 \times 10^{4}$ au, and the precession period is $1600 \pm 400$ years. The length of $\mathrm{CO}$ outflow is $\sim 120^{\prime \prime}$ in the $J=1 \rightarrow 0$ line (Section 3.2) and $\sim 100^{\prime \prime}$ in the $J=4 \rightarrow 3$ line (Davis et al. 2000), and there is no sign of outflow on a larger scale. Assuming that the length of the primary jet is the same as that of the CO outflow, the dynamical timescale of the V380 Ori NE outflow system is $\sim 6300$ years. This timescale may be a good estimate of the age of the protostar. In general, the outflow timescale has often been used to infer the age, but unreliable inclination estimates are a major source of ambiguity (Downes \& Cabrit 2007). In the case of the $\mathrm{SiO}$ outflow of V380 Ori NE, the combination of oscillation patterns of position angle and velocity allows an accurate estimation of the inclination angle, which resolves the ambiguity.

Rough estimates of protostellar mass can be made from the timescale and flow speed. Assuming a steady accretion with a "typical" accretion rate of $2 \times 10^{-6} M_{\odot} \mathrm{yr}^{-1}$ (Shu et al. 1987), the growth in mass during the outflow timescale is $\sim 0.013 \mathrm{M}_{\odot}$. If the protostar initially grew in a first hydrostatic core, the accretion rate would have been much higher (Saigo et al. 2008). This high-accretion phase may have lasted only for a relatively short time until the mass of the first core was exhausted, which was followed by the normal accretion phase. Assuming that the mass of the first core was $\sim 0.01 M_{\odot}$ (Saigo et al. 2008), the current mass of the protostar may be $\sim 0.02 M_{\odot}$. Alternatively, the outflow speed may be comparable to the Kepler speed on the surface of the protostar (Machida et al. 2008). Assuming that the protostar radius is in the range of $1-4 R_{\odot}$ (Machida et al. 2008; Masunaga \& Inutsuka 2000), a Kepler speed of $35 \mathrm{~km} \mathrm{~s}^{-1}$ suggests a stellar mass in the range of $0.006-0.03 M_{\odot}$. Therefore, $0.02 M_{\odot}$ may be a reasonable estimate of the protostellar mass.

The accretion luminosity can be calculated using the mass, accretion rate, and stellar radius in the previous paragraph. The expected luminosity is $0.3-1.2 L_{\odot}$. The luminosity of the protostar itself (from contraction and deuterium burning) can be ignored because the age is too young (Myers et al. 1998). By contrast, infrared observations of V380 Ori NE indicate $L_{\text {bol }}=3.9 L_{\odot}$ (Furlan et al. 2016). Considering the uncertainties and assumptions involved, it is unclear whether or not the difference between the expected and observed luminosities is significant. If it is, then this discrepancy suggests several possibilities: (1) the protostar may be more massive; (2) the system may be currently in a state of enhanced accretion; (3) there could be multiple protostars; or (4) the bolometric luminosity may be overestimated, considering that the infrared flux contains a contribution from the bright outflow knot. We use the protostellar mass of $0.02 M_{\odot}$ in the discussion below, but note that there is a possibility of larger mass.

The V380 Ori NE system is still extremely young, and the protostar may be evolving significantly over a jet precession period, which corresponds to about a quarter of the outflow timescale. For example, the mass may increase by more than $10 \%$ during a precession cycle. If the jet-driving protostar belongs to a binary system (Section 4.3), the matter accreting from the envelope may distribute the accompanying angular momentum into protostellar spins, disk rotation, and binary orbital motions. The quantities related to the jet precession, such as flow speed and precession period, then may also evolve at significant rates, and the oscillation pattern of the jet may be more complicated than the simple sinusoidal profiles described in Section 4.1. Indeed, the $\mathrm{CO} J=4 \rightarrow 3$ line map shows a change of outflow direction on a large scale (Davis et al. 2000). Davis et al. (2000) inferred an oscillation wavelength of $\sim 200^{\prime \prime}$ 


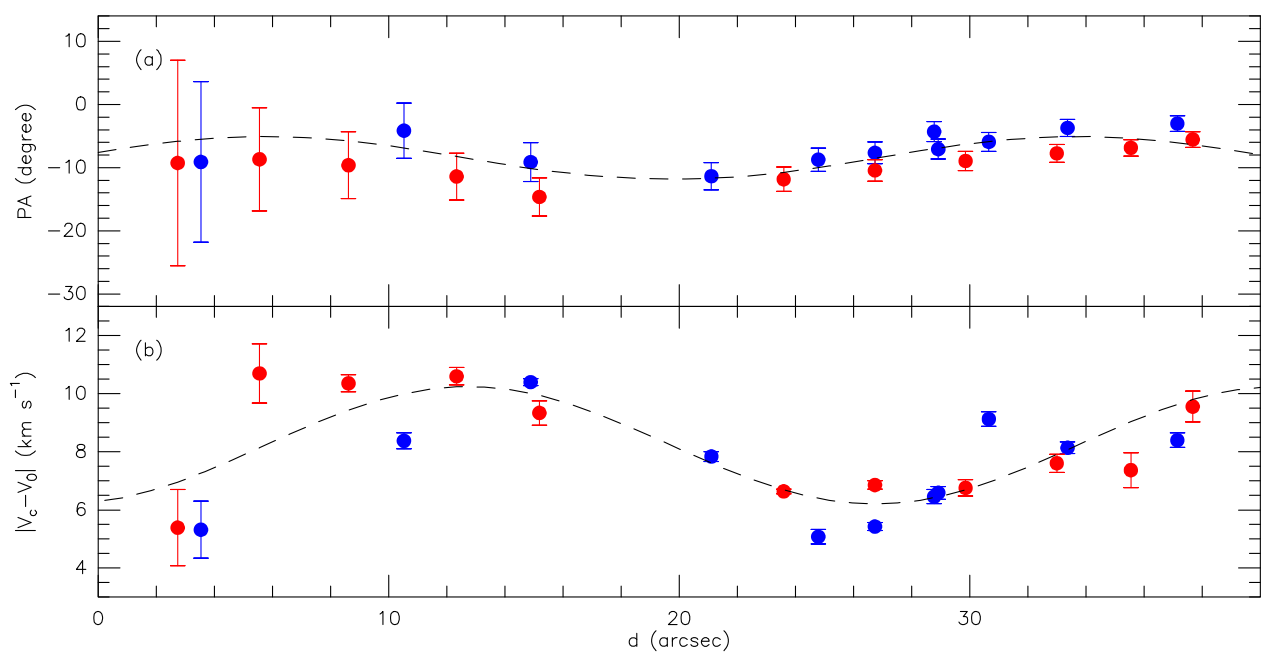

Figure 6. Parameters of the $\mathrm{SiO}$ emission peaks as functions of angular distance from the expected position of the protostar. Blue and red dots are for the northern and southern jets, respectively. (a) Position angle with respect to the protostellar position. For the southern jet, the plot shows the position angle minus $180^{\circ}$. Vertical bars show the angle subtended by the beam (1".6). (b) Centroid velocity with respect to the systemic velocity. Vertical bars show the uncertainty of the centroid velocity from the Gaussian fit to each spectrum. Dashed curves: best-fit sinusoidal functions.

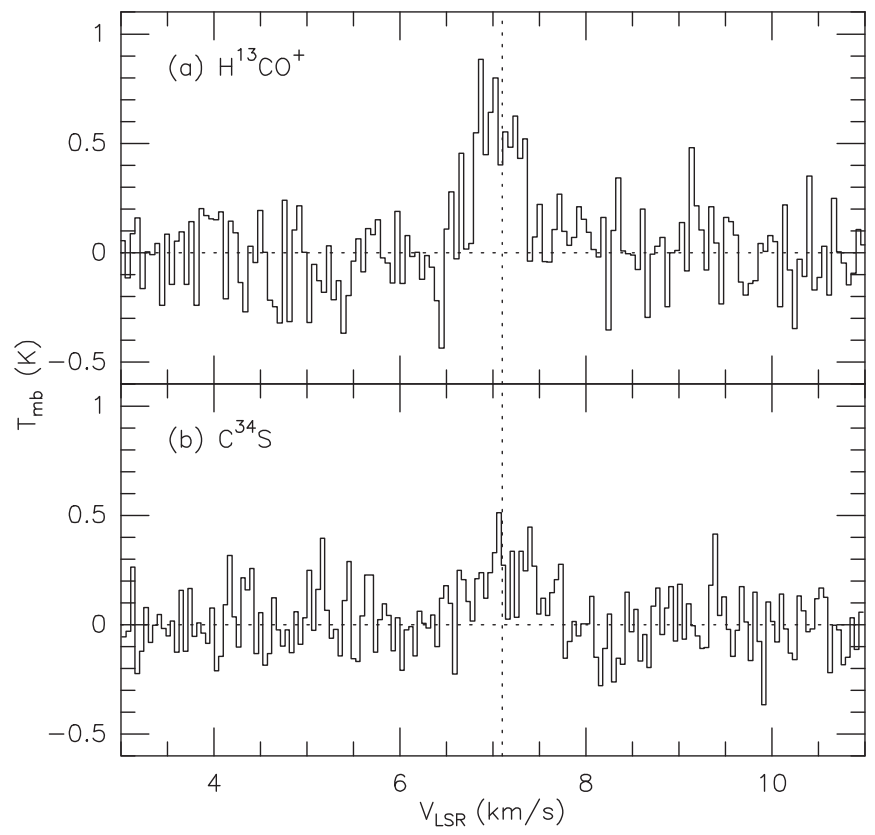

Figure 7. Spectra of (a) the $\mathrm{H}^{13} \mathrm{CO}^{+} J=1 \rightarrow 0$ line and (b) the $\mathrm{C}^{34} \mathrm{~S}$ $J=2 \rightarrow 1$ line toward the V380 Ori NE dense core. Vertical dotted line: $V_{\mathrm{LSR}}=7.1 \mathrm{~km} \mathrm{~s}^{-1}$.

for the $\mathrm{CO}$ outflow. If the $\mathrm{CO}$ and $\mathrm{SiO}$ outflows are tracing the same precession phenomenon, either the precession rate may be increasing or the flow speed may be decreasing. Alternatively, the outflow variability may be indicating a superposition of multiple precession modes.

\subsection{Precession Mechanism and Binary System}

The point-symmetric and oscillating pattern of the V380 Ori NE jet suggests that the jet axis (i.e., the rotation axis of jetlaunching mechanism) is precessing. The usual explanation for jet precession is that the jet-driving protostar belongs to a noncoplanar binary system, i.e., the jet-launching disk is misaligned with the binary orbital plane (Terquem et al. 1999; Bate et al. 2000; Sheikhnezami \& Fendt 2015). The disk responds to the tidal perturbation mainly in two

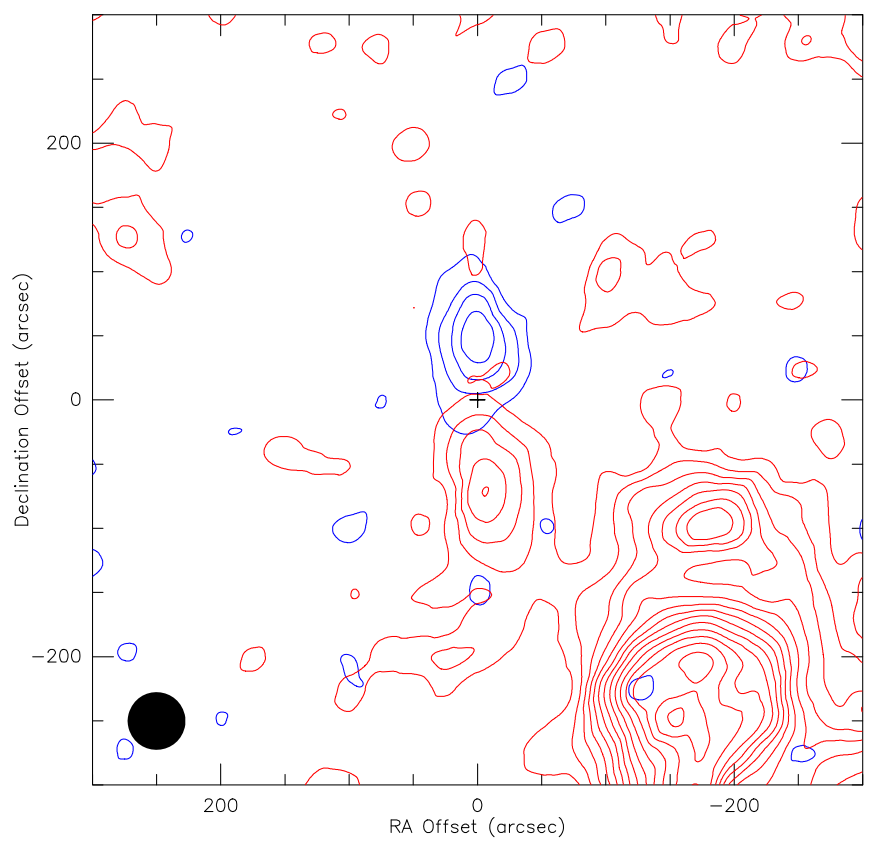

Figure 8. Maps of the CO $J=1 \rightarrow 0$ line toward V380 Ori NE. The blue and red contours show the $\mathrm{CO}$ line intensity integrated over the velocity ranges of $(-3.7,4.1)$ and $(10.0,20.4) \mathrm{km} \mathrm{s}^{-1}$, respectively. The lowest contour levels are $2 \mathrm{~K} \mathrm{~km} \mathrm{~s}^{-1}$ and $3 \mathrm{~K} \mathrm{~km} \mathrm{~s}^{-1}$ for blue and red contours, respectively. The contour interval is $2 \mathrm{~K} \mathrm{~km} \mathrm{~s}^{-1}$. Shown in the bottom left-hand corner is the beam: $\mathrm{FWHM}=45^{\prime \prime}$. Plus sign: the submillimeter continuum peak (Davis et al. 2000), which is the map center.

different modes: precession (response to the axially symmetric potential) and wobbling (relatively faster response to the azimuthal Fourier component of second harmonic).

First, the disk-jet system as a whole can precess. The precession period may be longer than the binary orbital period by an order of magnitude. Bate et al. (2000) suggested a factor of $\sim 20$ for a typical protostellar binary system. If the disk precesses for a period of 1600 years, the inferred orbital period is $\sim 80$ years. The separation between the binary members would then be $\sim 6$ au $\left(0{ }^{\prime \prime} 014\right.$ at $\left.418 \mathrm{pc}\right)$. Because the disk size should be smaller than the binary orbit, the disk radius cannot be larger than $\sim 2$ au. 
Table 3

Detected L1641 Continuum Sources

\begin{tabular}{|c|c|c|c|c|c|c|c|c|c|}
\hline \multirow{2}{*}{ Source } & \multirow{2}{*}{ Name } & \multicolumn{2}{|c|}{ Peak Position ${ }^{\mathrm{a}}$} & \multicolumn{3}{|c|}{ Flux Density ${ }^{\mathrm{b}}$} & \multicolumn{3}{|c|}{ Associated Objects ${ }^{c}$} \\
\hline & & $\alpha_{\mathrm{J} 2000}$ & $\delta_{\mathrm{J} 2000}$ & $1.4 \mathrm{~mm}$ & $1.1 \mathrm{~mm}$ & $\mathrm{~S} / \mathrm{N}$ & OrionAS & HOPS & TUKH \\
\hline 1 & L1641N MMS & 053618.8 & -062219 & 1.52 & 3.4 & 18.3 & 536196-62209 & $181-183$ & 67 \\
\hline 2 & Strom 11 & 053625.5 & -062452 & 0.63 & 1.7 & 7.1 & $536248-62454$ & $173-176,380$ & $\ldots$ \\
\hline 3 & TUKH 74 & 053641.0 & -062616 & 0.50 & 1.1 & 5.7 & $536414-62627$ & $\ldots$ & 74 \\
\hline 4 & HH 34 MMS & 053530.7 & -062653 & 0.58 & 1.3 & 5.8 & $535297-62701$ & $188-190$ & 71 \\
\hline 5 & V380 Ori NE & 053635.8 & -063854 & 0.76 & 1.4 & 8.3 & $536361-63857$ & $169(362)$ & 89 \\
\hline 6 & HH 147 MMS & 053625.5 & -064439 & 0.49 & 1.0 & 4.8 & $536259-64440$ & 166 & 91 \\
\hline 7 & HH 1-2 MMS 2 & 053618.6 & -064539 & 0.74 & 1.7 & 8.4 & 536179-64544 & 167,168 & $\ldots$ \\
\hline 8 & HH 1-2 MMS 1 & 053623.1 & -064609 & 0.70 & 1.7 & 8.5 & 536229-64618 & 165,203 & 92 \\
\hline
\end{tabular}

Notes.

a Units of right ascension are hours, minutes, and seconds, and units of declination are degrees, arcminutes, and arcseconds.

${ }^{\mathrm{b}}$ Peak flux densities in $\mathrm{Jy} \mathrm{beam}^{-1}$, and signal-to-noise ratio in the combined map of the 1.4 and $1.1 \mathrm{~mm}$ bands.

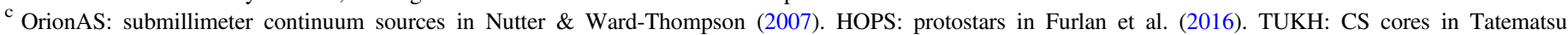
et al. (1993).

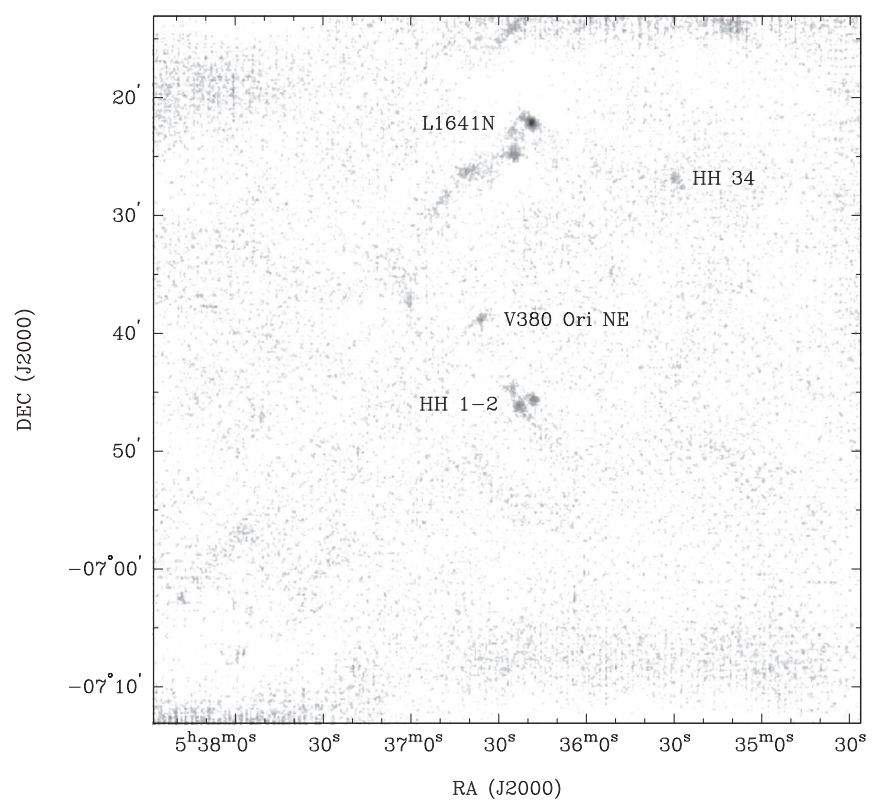

Figure 9. Combined image of the $\lambda=1.4$ and $1.1 \mathrm{~mm}$ continuum, showing the $60^{\prime} \times 60^{\prime}$ region of the MUSIC map. The maps of the two bands were averaged with a weighting to maximize the signal-to-noise ratio.

Second, the disk axis can wobble because of the tidal force, and the jet axis can precess along with the wobbling of the inner part of the disk (Bate et al. 2000). The period of wobble may be half the binary orbital period. From the jet precession period of 1600 years, the orbital period would be $\sim 3200$ years. The binary separation would be $\sim 70$ au ( 0 "' 17 at $418 \mathrm{pc})$, and the disk radius can be up to $\sim 20$ au.

The inferred binary separation provides an interesting insight into the binary formation process. In general, there are two channels for binary formation: fragmentation of the disk by gravitational instability and turbulent fragmentation of the dense cloud core. Theoretical models and observational imaging of binary systems suggest that the disk instability and turbulent fragmentation processes produce systems with binary separations smaller than $\sim 100$ au and larger than $\sim 500 \mathrm{au}$, respectively (Offner et al. 2010; Kratter \& Lodato 2016; Tobin et al. 2016; Lee et al. 2017). A survey of binary separations showed a bimodal distribution with peaks at $\sim 75$ au and $\sim 3000$ au, probably representing the disk instability and turbulent fragmentation modes of binary formation (Tobin et al. 2016). The binary separation of V380 Ori NE may be 6 or $70 \mathrm{au}$, depending on the precession mechanism. Either way, the separation suggests that this binary system was probably formed through the disk instability process.

There are alternative mechanisms of jet precession. For example, magnetically driven disk warping can cause the jet to precess (Lai 2003). In this mechanism, the disk is assumed to be perturbed by large-scale magnetic fields, and the protostar does not have to belong to a binary system.

Because the observed length of the $\mathrm{SiO}$ jet is only a little longer than a full precession cycle, the directional variability can be more complicated than the simple periodic precession. In such a case, alternative variability mechanisms are possible. For example, accretion of material from a turbulent cloud core can make the disk rotation axis change orientation chaotically (Bate et al. 2010; Tsukamoto \& Machida 2013). As a result, the jet axis can wander stochastically (Spalding et al. 2014). This turbulence-driven precession mechanism may be relevant to V380 Ori NE because the system is very young.

\subsection{Continuum Spectra of the L1641 Dense Cores}

SEDs of the detected cores are shown in Figure 14. Because the four wavelength bands were observed simultaneously, and then calibrated, imaged, and measured consistently, the spectral slopes based on the MUSIC data set are more reliable than those derived from inhomogeneous data sets. The detected cores span a wide range of spectral slope. When the flux density, $F_{\nu}$, is expressed as a power-law function of the frequency, $\nu$, the spectral index, $\alpha$ (i.e., $F_{\nu} \propto \nu^{\alpha}$ ), ranges from 2.0 to 3.9. The measured spectral slopes are consistent with that of thermal dust emission.

Before discussing the dust continuum emission further, it is necessary to consider possible contributions from other components of radiation. In particular, V380 Ori NE, HH 34 MMS, and HH 147 MMS show spectral slopes relatively shallower than the others, and "contaminations" in the long wavelength bands need to be examined. First, molecular lines in the continuum bands can affect the spectral slope. Practically, only very bright $\mathrm{CO}$ lines or maser lines can cause noticeable effects. The MUSIC 1.4 and $0.89 \mathrm{~mm}$ bands include the $\mathrm{CO} J=2 \rightarrow 1$ and $3 \rightarrow 2$ lines, respectively. Even if these 


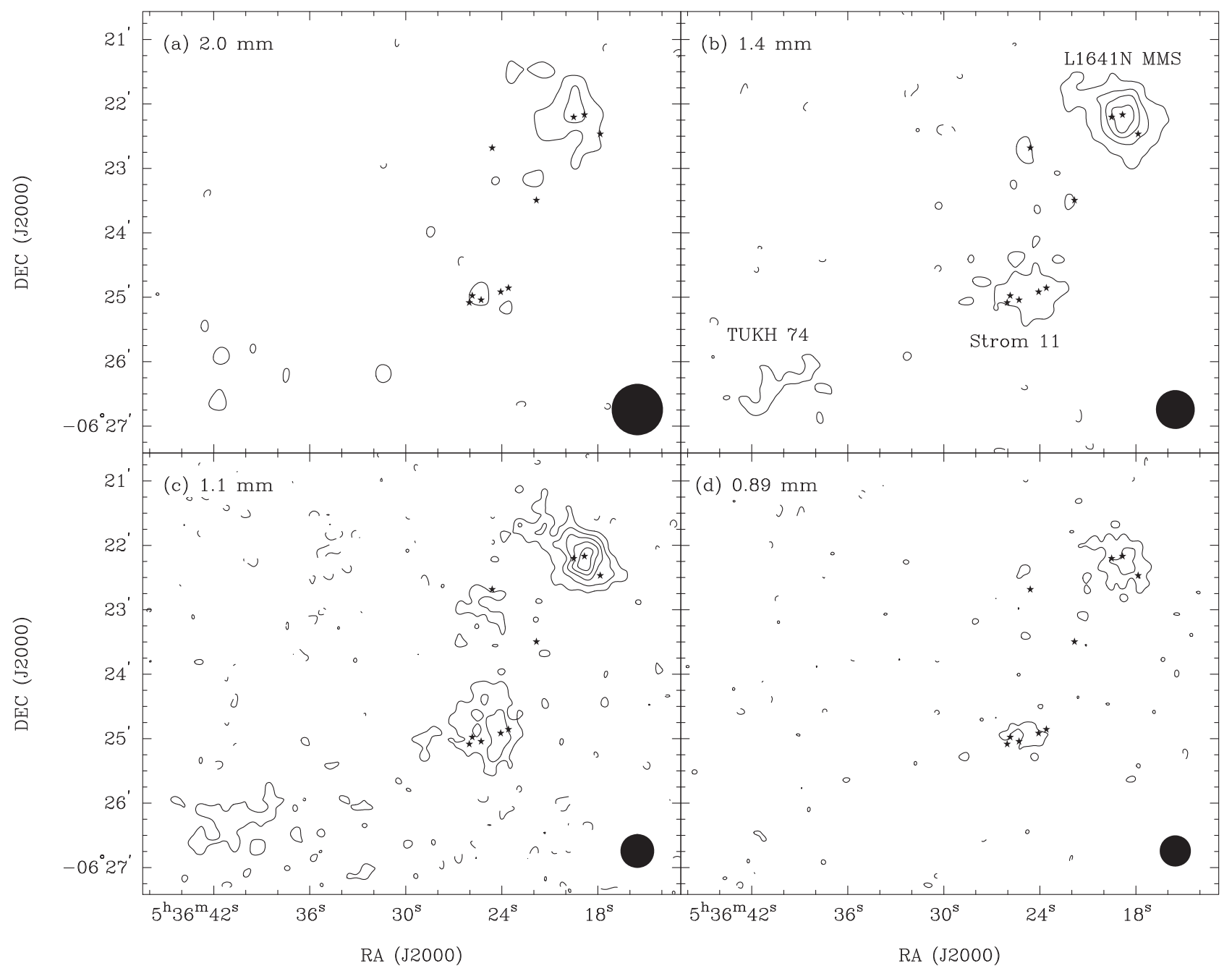

Figure 10. Maps of the $\mathrm{L} 1641 \mathrm{~N}$ region in the (a) $2.0 \mathrm{~mm}$, (b) $1.4 \mathrm{~mm}$, (c) $1.1 \mathrm{~mm}$, and (d) $0.89 \mathrm{~mm}$ continuum emission. The contour levels are $1,2,3,4$, and $5 \times 3 \sigma$, where $\sigma$ is the noise rms level of each map (Table 2). Dashed contours are for negative levels. Shown in the bottom right-hand corner of each panel is the beam size (Table 2). Detected continuum sources are labeled. Star symbols: protostars in the HOPS catalog (Furlan et al. 2016).



Figure 11. Maps of the HH 34 region in the millimeter-submillimeter continuum emission. See Figure 10 for the contour levels and markers.

bands contain extra flux densities from the CO lines, they would not necessarily make the slope shallower. The contamination by CO, especially the $J=3 \rightarrow 2$ line, would steepen the spectral slope and cannot be responsible for the shallow slopes. Moreover, the three shallow-spectrum cores were undetected in the $0.89 \mathrm{~mm}$ image, suggesting that the $\mathrm{CO}$ $J=3 \rightarrow 2$ line is too weak to affect the spectral slope. There is no strong maser line in the MUSIC bands. Therefore, molecular lines are unlikely to affect the continuum spectra. Second, some emission components can affect the long wavelength bands. The free-free emission may have a flat spectrum in the millimeter range, and the anomalous microwave emission may have a spectrum peaking at $\sim 25 \mathrm{GHz}$ and quickly decreasing at higher frequencies. The $3.6 \mathrm{~cm}$ flux density of the $\mathrm{HH} 34$ radio jet is $0.16 \mathrm{mJy}$ (Rodríguez \& Reipurth 1996), V380 Ori NE was undetected at $6 \mathrm{~cm}$ 


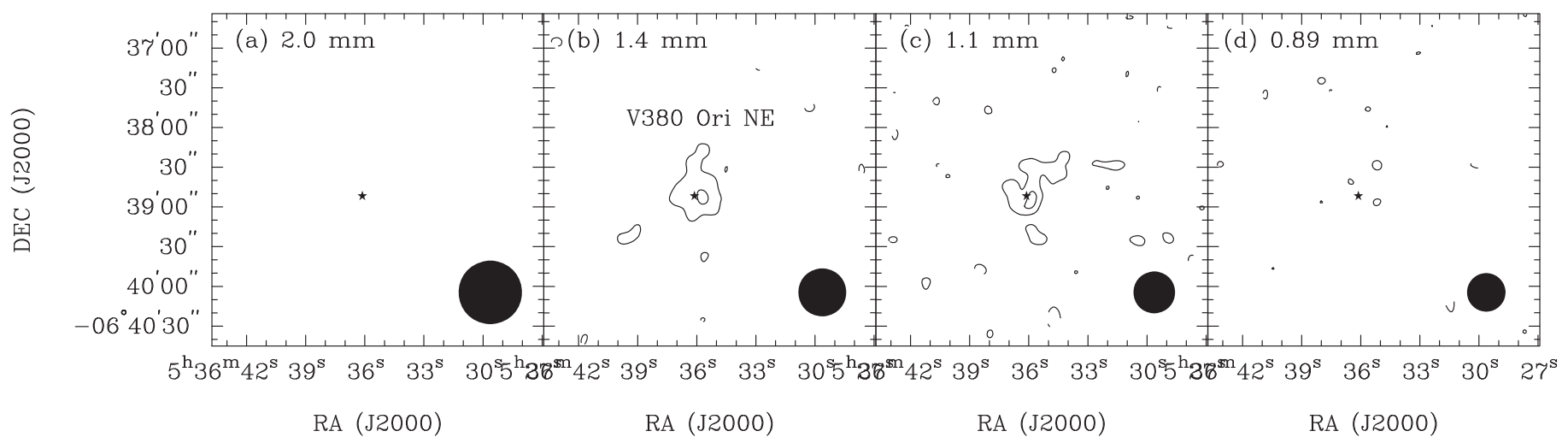

Figure 12. Maps of the V380 Ori NE region in the millimeter-submillimeter continuum emission. See Figure 10 for the contour levels and markers.



Figure 13. Maps of the HH 1-2 region in the millimeter-submillimeter continuum emission. See Figure 10 for the contour levels and markers.

Table 4

L1641 Continuum Source Parameters

\begin{tabular}{|c|c|c|c|c|c|c|c|}
\hline \multirow{2}{*}{ Name } & \multirow{2}{*}{$R_{A}{ }^{\mathrm{a}}$} & \multicolumn{4}{|c|}{ Flux Density ${ }^{\mathrm{b}}$} & \multirow{2}{*}{$\beta^{\mathrm{c}}$} & \multirow{2}{*}{$\begin{array}{c}\operatorname{Mass}^{\mathrm{c}} \\
\left(M_{\odot}\right)\end{array}$} \\
\hline & & $2.0 \mathrm{~mm}$ & $1.4 \mathrm{~mm}$ & $1.1 \mathrm{~mm}$ & $0.89 \mathrm{~mm}$ & & \\
\hline $\mathrm{L1641N} \mathrm{MMS}^{\mathrm{d}}$ & $60^{\prime \prime}$ & $1.07 \pm 0.20$ & $3.04 \pm 0.52$ & $6.4 \pm 1.2$ & $19.9 \pm 6.2$ & $1.4 \pm 0.3$ & $11.6_{-3.9}^{+5.8}$ \\
\hline Strom 11 & $48^{\prime \prime}$ & $0.31 \pm 0.10$ & $1.34 \pm 0.21$ & $4.1 \pm 0.6$ & $11.1 \pm 2.2$ & $2.2 \pm 0.3$ & $16.9_{-6.1}^{+9.5}$ \\
\hline TUKH 74 & $51^{\prime \prime}$ & $0.42 \pm 0.10$ & $1.12 \pm 0.29$ & $3.0 \pm 0.4$ & $5.7 \pm 1.5$ & $1.4 \pm 0.3$ & $4.3_{-1.5}^{+2.3}$ \\
\hline HH 34 MMS & $48^{\prime \prime}$ & $0.41 \pm 0.12$ & $0.73 \pm 0.11$ & $1.9 \pm 0.4$ & $\ldots$ & $0.7 \pm 0.6$ & $1.0_{-0.6}^{+1.4}$ \\
\hline V380 Ori NE & $36^{\prime \prime}$ & $\ldots$ & $0.71 \pm 0.11$ & $1.4 \pm 0.2$ & $\ldots$ & $0.3 \pm 0.5$ & $0.4_{-0.2}^{+0.4}$ \\
\hline HH 147 MMS & $40^{\prime \prime}$ & $\ldots$ & $0.67 \pm 0.19$ & $1.2 \pm 0.3$ & $\ldots$ & $0.7 \pm 0.7$ & $0.8_{-0.5}^{+1.1}$ \\
\hline HH 1-2 MMS 2 & $38^{\prime \prime}$ & $\ldots$ & $0.95 \pm 0.11$ & $2.7 \pm 0.3$ & $6.0 \pm 1.0$ & $1.9 \pm 0.4$ & $6.7_{-2.6}^{+4.1}$ \\
\hline HH 1-2 MMS 1 & $48^{\prime \prime}$ & $0.43 \pm 0.11$ & $1.30 \pm 0.23$ & $3.7 \pm 0.6$ & $7.9 \pm 2.0$ & $1.6 \pm 0.3$ & $6.6_{-2.3}^{+3.6}$ \\
\hline
\end{tabular}

Notes.

${ }^{a}$ Radius of the circular aperture for flux measurements.

${ }^{\mathrm{b}}$ Integrated flux densities in Jy.

${ }^{\mathrm{c}}$ Dust emissivity index and mass of molecular gas, assuming $T_{d}=20 \mathrm{~K}$.

d Parameters from Table 5 method E + A.

$(<0.1 \mathrm{mJy}$; Morgan et al. 1990), and HH 147 MMS was undetected at $6 \mathrm{~cm}$ or $3.6 \mathrm{~cm}(<0.03 \mathrm{mJy} ;$ Rodríguez et al. 2000). Therefore, the long-wavelength emission components are negligible in the millimeter range. Finally, external heating by the interstellar radiation field can increase the flux densities in the long wavelength bands, though the effect on the spectral slope may be small. This effect may be noticeable only for low-luminosity objects $\left(<1 L_{\odot}\right)$ in extremely strong radiation fields (Shirley et al. 2002; Furlan et al. 2016). Because the bolometric luminosities of HH 34 MMS, V380 Ori $\mathrm{NE}$, and HH 147 MMS are $L_{\text {bol }}=18.8,3.9$, and $15.5 L_{\odot}$, respectively (Furlan et al. 2016), it is unlikely that the external heating can affect the spectral slopes. Therefore, the millimeter-submillimeter flux densities of the detected cores are dominated by thermal dust emission (see further discussions in the last paragraph of Section 4.5).

Most of the dust continuum emission in the MUSIC beams may come from the dense cores, and the accretion disks around embedded protostars may contribute only a little. Interferometric observations with sub-arcseconds resolutions are needed to measure the flux densities from the disks, but there is no such observation available for the detected cores. Stanke \& Williams (2007) observed L1641N MMS with a $\sim 1$ !"4 resolution, and detected a compact source, L1641N MM1, 


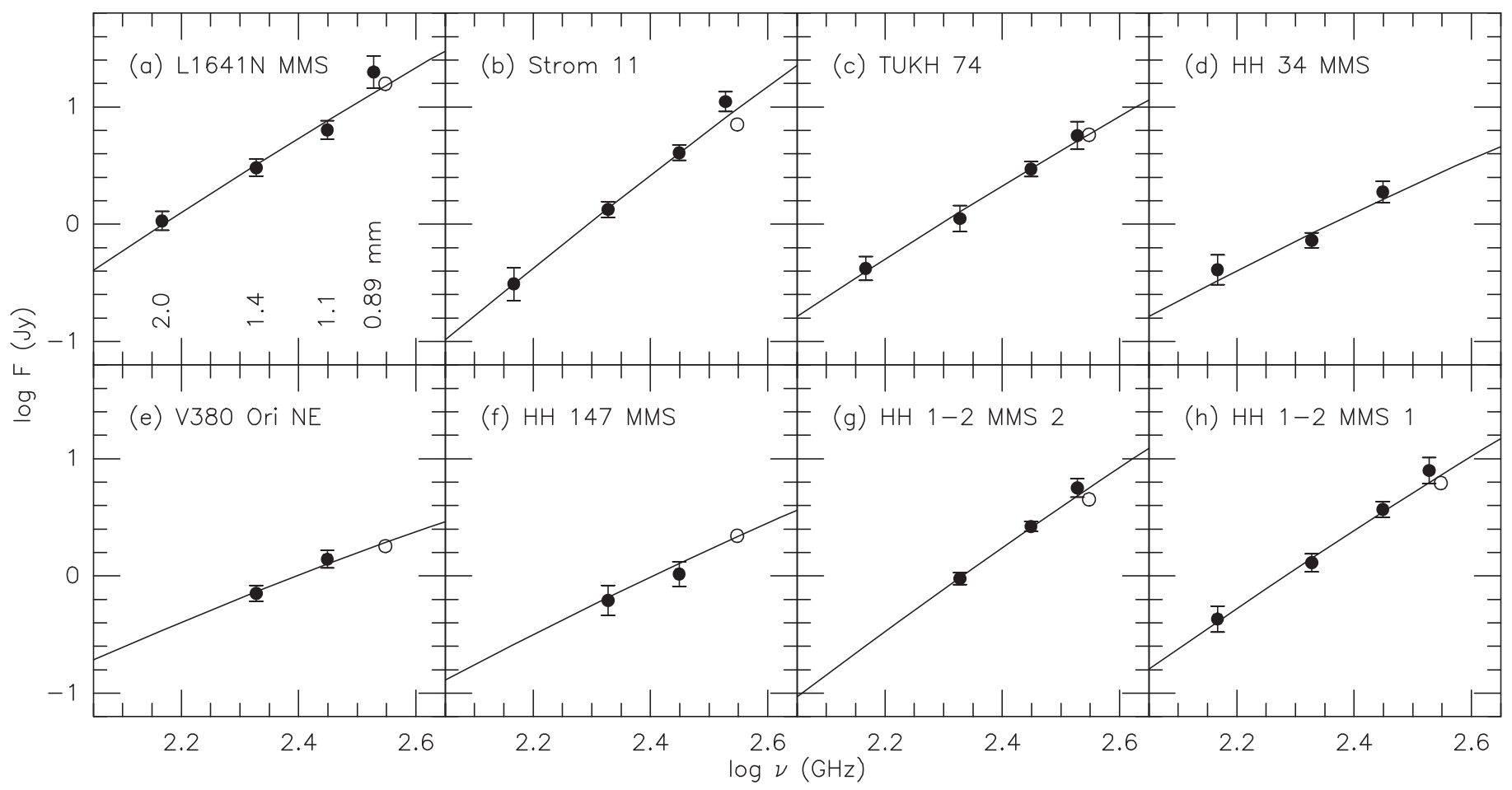

Figure 14. Spectral energy distributions of the detected dense cores. Filled circles: flux densities from the MUSIC maps. Flux uncertainties (1 $\sigma$ ) are shown. Open circles: $850 \mu \mathrm{m}$ flux densities from Nutter $\&$ Ward-Thompson (2007). Solid curves: best-fit modified black body spectra, assuming $T_{d}=20 \mathrm{~K}$.

Table 5

L1641N MMS Photometry

\begin{tabular}{lccrr}
\hline \hline \multirow{2}{*}{ Method $^{\mathrm{a}}$} & \multicolumn{3}{c}{ Flux Density $^{\mathrm{b}}$} & \multicolumn{1}{c}{$\beta$} \\
\cline { 2 - 5 } & $2.0 \mathrm{~mm}$ & $1.4 \mathrm{~mm}$ & $1.1 \mathrm{~mm}$ & $0.89 \mathrm{~mm}$ \\
\hline $\mathrm{U}+\mathrm{A}$ & $1.05 \pm 0.20$ & $2.94 \pm 0.50$ & $6.2 \pm 1.1$ & $19.0 \pm 5.9$ \\
$\mathrm{E}+\mathrm{A}$ & $1.07 \pm 0.20$ & $3.04 \pm 0.52$ & $6.4 \pm 1.2$ & $19.9 \pm 6.2$ \\
$\mathrm{E}+\mathrm{G}$ & $1.17 \pm 0.26$ & $2.82 \pm 0.39$ & $6.6 \pm 1.1$ & $1.4 \pm 0.3$ \\
\hline
\end{tabular}

Notes.

${ }^{\mathrm{a}} \mathrm{U}$ : The correction for the effect of high-pass filter was made using $\eta_{\mathrm{hpf}}$ for an unresolved source, listed in Table 2. E: The correction was made using $\eta_{\mathrm{hpf}}=0.87$, $0.89,0.90$, and 0.90 , which is appropriate for the size of L1641N MMS. A: The flux density was integrated in a circular aperture. G: The peak flux density was measured by fitting the image with an elliptical Gaussian profile, and scaled for the solid angle of the source.

${ }^{\mathrm{b}}$ Integrated flux densities in Jy.

with $0.54 \mathrm{Jy}$ at $1.3 \mathrm{~mm}$. They interpreted this source as an envelope structure rather than a disk. Thus, the contribution of the disk to the MUSIC flux density must be much smaller than $\sim 10 \%$. Even so, the disk in L1641N MM1 is presumably one of the brightest examples in the observed region. Therefore, the contribution from the protostellar disks in the detected cores is unimportant in the interpretation of the SEDs from MUSIC data.

\subsection{Dust Emissivity and Mass of Dense Cores}

The dust continuum radiation is expected to be optically thin in the wavelength range covered. The mass emissivity is often expressed in a power-law function of the frequency

$$
\kappa_{\nu}=0.1\left(\frac{\nu}{\nu_{0}}\right)^{\beta} \mathrm{cm}^{2} \mathrm{~g}^{-1},
$$

where $\nu_{0}=1 \mathrm{THz}$, and $\beta$ is the emissivity index (Hildebrand 1983; Henning et al. 1995; André et al. 2010; Sadavoy et al. 2016). This equation implicitly assumes a gas-to-dust mass ratio of $\sim 100$. The emissivity in the millimeter range is extrapolated from $1 \mathrm{THz}$ because the emissivity is known better in the infrared range (Henning et al. 1995).

The SED can be fitted with a modified blackbody function,

$$
F_{\nu}=\frac{M}{D^{2}} \kappa_{\nu} B_{\nu}\left(T_{d}\right),
$$

where $M$ is the mass of molecular gas, $D$ is the distance to the source, $B_{\nu}$ is the Planck function, and $T_{d}$ is the dust temperature. In the millimeter wavelength range, the SED fit is almost insensitive to $T_{d}$, and $\beta$ depends on $T_{d}$ only weakly. A typical temperature for dense cores is $T_{d}=20 \mathrm{~K}$ (Johnstone \& Bally 2006). Specifically, Stutz et al. (2013) derived $20.4 \mathrm{~K}$ for V380 Ori NE by fitting the SED in the $70-870 \mu \mathrm{m}$ range.

SED fits to the measured flux densities were made assuming $T_{d}=20 \mathrm{~K}$. Four cores were detected at $0.89 \mathrm{~mm}$, and their flux densities are consistent with the $850 \mu \mathrm{m}$ flux densities reported 






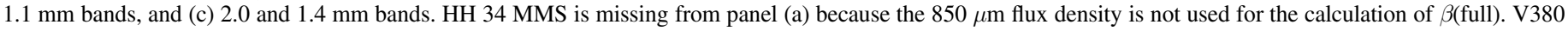
Ori NE, HH 147 MMS, and HH 1-2 MMS 2 are missing from panels (b) and (c) because they were undetected in the 2.0 mm band.

by Nutter \& Ward-Thompson (2007). Therefore, the MUSIC data and the $850 \mu \mathrm{m}$ data were used together for the SED fits. For the purpose of weighting, the uncertainty of the $850 \mu \mathrm{m}$ data were set to be $20 \%$, which gives weights roughly similar to those of the MUSIC data points. The $850 \mu \mathrm{m}$ flux density was not used for HH 34 MMS because there is a secondary source (IRS 5) near the edge of the main source, which makes it difficult to treat the data consistently.

Figure 14 shows the best fits, and Table 4 lists the derived $\beta$. The single-component fits are reasonably good, and most data points agree with the fits within the uncertainties. The average $\beta$ is 1.3 , which is in the range of typical $\beta$ values of dense cores (Section 4.6). V380 Ori NE, HH 34 MMS, and HH 147 MMS have particularly lower $\beta$ values $(0.3,0.7$, and 0.7 , respectively) than the other cores, suggesting that they contain a considerable amount of large dust grains. Masses of the cores were derived from the SED fits (Table 4), assuming $D=418$ pc (Kim et al. 2008). The core masses in the literature are often calculated using a fixed value of emissivity at a certain frequency. For example, Nutter \& Ward-Thompson (2007) assumed $0.01 \mathrm{~cm}^{2} \mathrm{~g}^{-1}$ at $850 \mu \mathrm{m}$, which corresponds to $\beta \approx 2.2$ in Equation (6). For the three cores with relatively high $\beta$ (Strom 11, HH 1-2 MMS 2, and HH 1-2 MMS 1), the mass estimates in this paper agree with those of Nutter \& Ward-Thompson (2007) within 40\%. For the three cores with low $\beta$ (V380 Ori NE, HH 34 MMS, and HH 147 MMS), the masses in Table 4 are smaller than those of Nutter \& WardThompson (2007) by a factor of $\sim 4$. Note that the mass estimates given here critically depend on the emissivity assumed in Equation (6), and it can be a major source of uncertainty. For example, the proportionality constant can be different if the cores have non-standard gas-to-dust mass ratios. The simple power-law description can also be an issue, because the index $\beta$ is not necessarily a constant over a large range of frequency (Draine 2006; Coupeaud et al. 2011).

While the inclusion of the $850 \mu \mathrm{m}$ data reduces the uncertainty in $\beta$, especially for the sources undetected in the $0.89 \mathrm{~mm}$ band, it makes the data set inhomogeneous. There is a possibility of bias coming from the differences in calibration, filtering, and photometry. A more aggressive filtering of the $850 \mu \mathrm{m}$ data can bias $\beta$ toward lower values. To understand the effect of the $850 \mu \mathrm{m}$ data, the emissivity index was calculated using the 1.4 and $1.1 \mathrm{~mm}$ band data and compared with that of the full data set. Figure 15(a) shows that there is no obvious bias. The range of $\beta$ values and the order among the sources are largely unchanged. For V380 Ori NE and HH $147 \mathrm{MMS}$, the fits to the 1.4 and $1.1 \mathrm{~mm}$ band data give $\beta=0.7 \pm 0.8$ and $0.5 \pm 1.4$, respectively. Though these emissivity indices are still less than one, the uncertainties are large. The low $\beta$ values of these cores are, at most, very tentative, and consequently the discussion given below (on the pre-protostellar core lifetime) is also tentative.

The emissivity index from the SED fit has a weak dependency on the dust temperature assumed. In the Rayleigh-Jeans (high temperature) limit, the $\beta$ value would be lower by 0.3 than that of $T_{d}=20 \mathrm{~K}$. A lower $T_{d}$ gives a higher $\beta$ value. Radiative transfer models of protostellar envelope show that the dust temperature decreases outward from the center, hits a minimum temperature of $\sim 10 \mathrm{~K}$, and increases again near the edge of the cloud, owing to the heating by the interstellar radiation field (Shirley et al. 2002). The $\beta$ value from the SED fit assuming $T_{d}=10 \mathrm{~K}$ can then be considered as an upper limit. The limits for some of the detected cores are: $\beta<2.6$ for Strom 11, $\beta<1.1$ for HH $34 \mathrm{MMS}$, and $\beta<0.7$ for V380 Ori NE.

To test the presence of any extra emission component, the emissivity index was calculated using subsets of the MUSIC data and compared with that of the full data set. Figure 15(b) shows $\beta$ (subset) from the 2.0 and $1.1 \mathrm{~mm}$ bands. These bands do not contain the $\mathrm{CO}$ lines. All the data points are consistent with $\beta$ (subset $)=\beta$ (full) within the uncertainties, which suggests that there is no noticeable effect of CO lines on the spectral slope. Figure 15(c) shows the $\beta$ (subset) from the 2.0 and $1.4 \mathrm{~mm}$ bands. If there were a significant contribution from long-wavelength emission components, such as free-free or anomalous microwave emission, the corresponding source would show $\beta$ (subset) $<\beta$ (full). Most of the data points are located near the $\beta$ (subset) $=\beta$ (full) line, which suggests that there is no significant contamination from long-wavelength emission components. The lowest data point (corresponding to HH 34 MMS) is located marginally below the line. There are a few possible explanations. First, the $2.0 \mathrm{~mm}$ flux density may be very uncertain and somehow overestimated. Second, the two sub-cores (Appendix) may have different dust properties, and the single-component fit may not work well. Third, there may 
be a long-wavelength emission component. Though the radio jet is not strong enough to affect the millimeter spectrum (Section 4.4), a low-level diffuse free-free emission component (large enough to be resolved out by VLA) could contaminate the $2.0 \mathrm{~mm}$ flux density. For HH $34 \mathrm{MMS}$, the fit to the 1.4 and $1.1 \mathrm{~mm}$ band data gives $\beta=1.8 \pm 0.9$. Therefore, the low $\beta$ value of HH 34 MMS is very tentative.

\subsection{Emissivity Index and Other Physical Quantities}

The low $\beta$ values of V380 Ori NE, HH $34 \mathrm{MMS}$, and HH 147 MMS suggest that dust grains can grow to a large size in some dense cores. Microscopically, the size, composition, and structure of dust can affect emissivity index, and growth of ice mantles and coagulation of particles may be the important physical processes in dense cores (Ossenkopf \& Henning 1994; Henning et al. 1995; Ormel et al. 2009). Observationally, submillimeter studies of nearby star-forming regions showed that dense cores usually have $\beta=1.0-2.0$ (e.g., Visser et al. 1998; Hogerheijde \& Sandell 2000). Recent studies using the data in the far-infrared to submillimeter range showed that dense cores have $\beta=1.0-2.7$ (Sadavoy et al. 2016; Chen et al. 2016). In the diffuse interstellar medium, molecular-gas dominated regions have $\beta \approx 1.66$ (Planck Collaboration et al. 2014). In these studies, however, the emissivity index may have dependencies on the dust temperature. Sadavoy et al. (2013) suggested that data in long wavelengths are important. Schnee et al. (2014) reported $\beta=0.6$ for OMC 3 MMS 6, but their low $\beta$ estimates were later disputed by Sadavoy et al. (2016). Therefore, the low $\beta$ values of the three cores in this study are rather unusual.

Because the emissivity index spans a wide range, it would be interesting to examine relations with other physical quantities that are likely relevant to dust properties. Figure 16 shows the correlations of the emissivity index with the properties of protostars embedded in the dense cores. The multiplicity, $N_{\mathrm{ps}}$, in Figure 16(e) is the number of protostars in the radii given in Table 4. The correlations with a kinematic property of the molecular gas, the widths of molecular lines tracing dense gas, are shown in Figure 17.

Properties of protostars have only weak correlations with $\beta$, if any. It is unclear if there is a positive correlation with the bolometric luminosities (Figures 16(a), (b)), and the linear correlation coefficient is $r \approx 0.3$. The lack of correlation with bolometric temperatures $(r \approx 0.1$; Figures $16(\mathrm{c}),(\mathrm{d}))$ is surprising because the variations in dust properties are often considered to be evolutionary effects (Visser et al. 1998; Dent et al. 1998). Froebrich (2005) also found that there is no correlation between $\beta$ and $L_{\mathrm{bol}}$ or $T_{\mathrm{bol}}$. There is a weak correlation with the number of protostars $(r=0.5$; Figure 16(e)), but more data are needed to confirm this correlation.

The strongest correlation with $\beta$ comes from the molecular line widths (Figure 17). The linear correlation coefficients are $r=0.6,0.7$, and 0.7 for $\mathrm{CS}, \mathrm{C}^{18} \mathrm{O}$, and $\mathrm{NH}_{3}$, respectively. This correlation suggests that turbulence may be a major factor affecting dust properties, though the exact mechanism is unclear. The turbulence in dense cores may be related to cloud support and fragmentation on large scales and the motions of dust particles on small scales. In turn, turbulence may be affecting the global timescale and also the local grain size distribution. It has been suggested that grain dynamics may be a major factor in the shattering and coagulation of dust grains
(Chokshi et al. 1993; Hirashita \& Yan 2009; Ormel et al. 2009). Another relevant process may be the cloud-scale clustering of dynamically decoupled grains (Hopkins 2014; Hopkins \& Lee 2016).

\subsection{Timescales and Dust Growth in Dense Cores}

Dust grains can grow to a large size in the circumstellar structure around relatively evolved YSOs, such as protoplanetary disks (Testi et al. 2014). It has been unclear whether or not dense cores or protostellar envelopes contain large dust grains, and if so, when they start to grow such grains. Kwon et al. (2009) reported that some Class 0 protostars have $\beta$ values around or smaller than 1 , based on interferometric observations at 1.3 and $2.7 \mathrm{~mm}$ wavelength bands. They suggested that the central $\sim 400$ au region of the protostellar envelope contains large grains, up to $\sim 1 \mathrm{~cm}$. Miotello et al. (2014) suggested the presence of millimeter-sized dust grains in the envelopes of Class I protostars. Large dust grains in protostellar envelopes are interesting because they can behave like a separate fluid component and cause intriguing effects, such as the increase of dust-to-gas ratio in the central region, spatial segregation of grains by size, and formation of flattened dust-rich structure (Bate \& Lorén-Aguilar 2017). This flattened structure can have a radius of several hundreds of au and is probably observable using radio interferometers.

Ormel et al. (2009) simulated the evolution of dust population in dense cores. They found that grain growth is negligible in free-fall timescales $\left(\sim 10^{5}\right.$ years for a density of $10^{5} \mathrm{~cm}^{-3}$ ) but becomes significant on larger timescales, such as ambipolar diffusion timescale $\left(\sim 4 \times 10^{6}\right.$ years for a density of $10^{5} \mathrm{~cm}^{-3}$ ). Wong et al. (2016) investigated the dust coagulation process in protostellar envelopes and found that the grain growth at a density of $10^{5} \mathrm{~cm}^{-3}$ is not fast enough to produce millimeter-sized grains. They suggested that such grains may be formed in the central dense $\left(10^{10} \mathrm{~cm}^{-3}\right.$ or higher) region of the envelope and transported outward by the protostellar outflow, up to a distance of 1000 au in $10^{4}$ years.

A $\beta$ value lower than 1 implies that the source contains dust grains up to a few times the wavelength observed (Draine 2006), and the low $\beta$ values of V380 Ori NE, HH 34 MMS, and $\mathrm{HH} 147 \mathrm{MMS}$ suggest that these cores, or envelopes of Class 0-I protostars, contain dust grains as large as several millimeters. In the porous composite grain model of Ricci et al. (2010), $\beta \approx 0.3$ implies a maximum grain size of at least $\sim 7$ $\mathrm{mm}$. If these large grains are mainly icy grains, $\beta \approx 0.3$ implies a maximum grain size of $\sim 2 \mathrm{~cm}$ or larger (see Figure 4 of Testi et al. 2014). For these three cores, the size of the $850 \mu \mathrm{m}$ emission sources is larger than the $\sim 15^{\prime \prime}$ beam (Nutter $\&$ WardThompson 2007). Therefore, these cores seem to contain millimeter-sized grains in $\sim 10,000$ au scale regions.

The outflow-transportation model of Wong et al. (2016) cannot explain the presence of the large grains in the V380 Ori $\mathrm{NE}$ and HH 34 MMS cores, because the ages of the protostars in these cores are not old enough for the growth of millimetersized grains at the central region and the subsequent transportation to a distance of $\sim 10,000 \mathrm{au}$. The large grains should have been formed in the observed volume of the core. The case of HH 147 MMS is less clear because the protostar $\left(\mathrm{N}^{3} \mathrm{SK} 50\right)$ is relatively evolved (Appendix). The timescale issue is especially acute in the case of V380 Ori NE. This core contains a single protostar (or a protobinary), and its outflow 


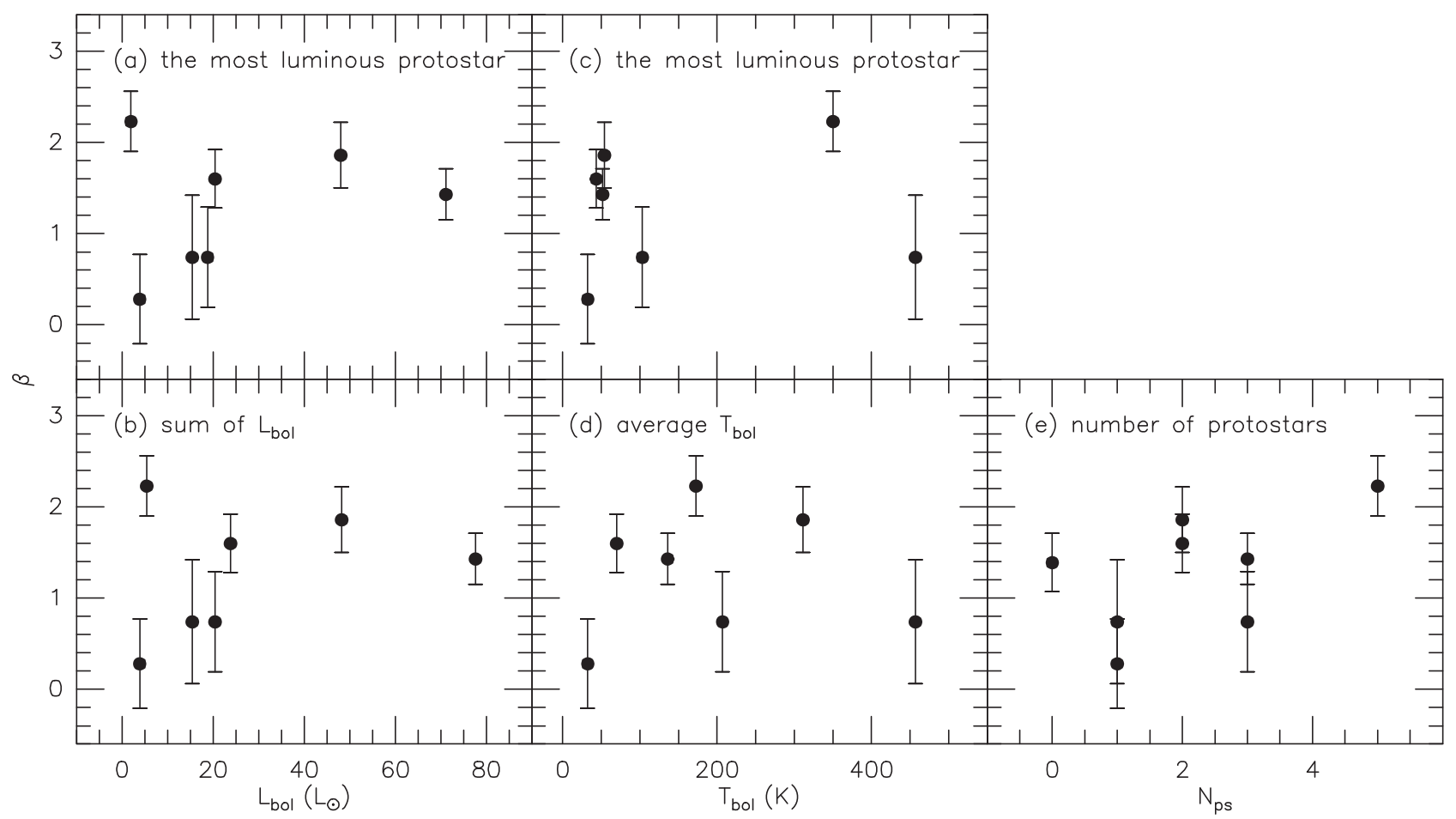

Figure 16. Correlation diagrams of the dust emissivity index with various properties of the dense cores: (a) bolometric luminosity of the most luminous protostar, (b) sum of the bolometric luminosities of the protostars, (c) bolometric temperature of the most luminous protostar, (d) average bolometric temperature of the protostars, and (e) number of protostars in the core. The bolometric luminosities and temperatures are from Furlan et al. (2016). TUKH 74 is missing from panels (a-d) because it contains no protostar.

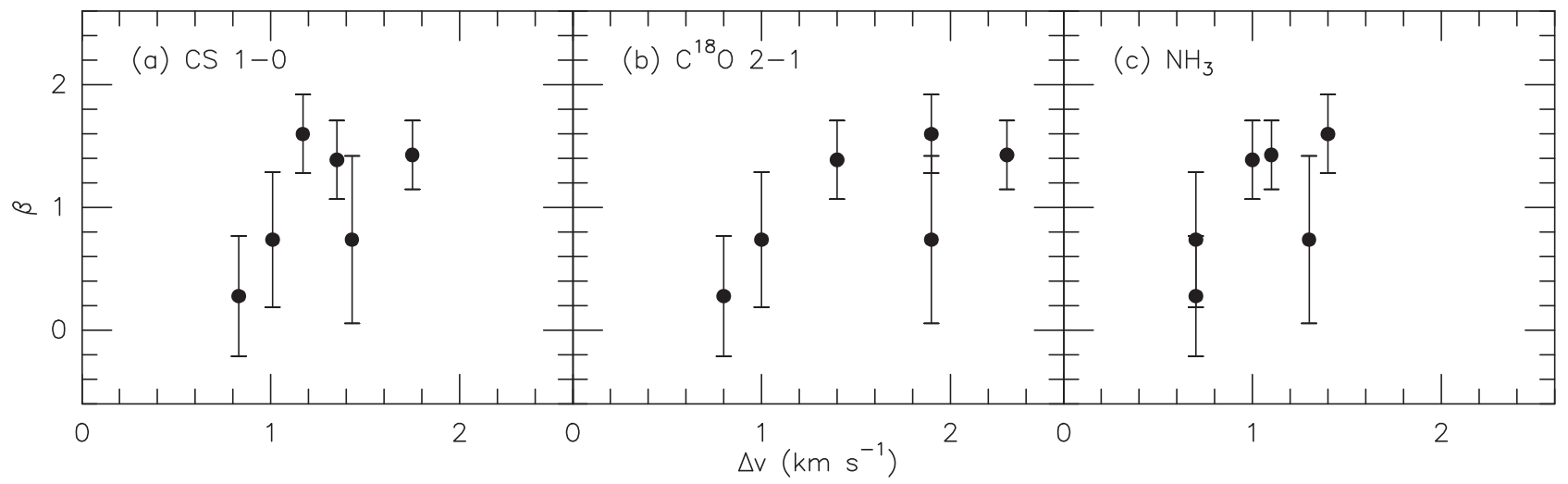

Figure 17. Correlation diagrams of the dust emissivity index with molecular line widths: (a) $\mathrm{CS} J=1 \rightarrow 0$ (Tatematsu et al. 1993), (b) $\mathrm{C}^{18} \mathrm{O} J=2 \rightarrow 1$ (Wilson et al. 1999), and (c) $\mathrm{NH}_{3}(1,1) /(2,2)$ (Wilson et al. 1999). Strom 11 and $\mathrm{HH} 1-2 \mathrm{MMS} 2$ are missing from these plots because there is no counterpart in the CS core catalog of Tatematsu et al. (1993).

timescale is only $\sim 6300$ years (Section 4.2 ), which is much shorter than the free-fall timescale. Since the age of the protostar may be comparable to the outflow timescale, the large grains should have been present already at the onset of the protostellar collapse. To grow these large grains, the V380 Ori $\mathrm{NE}$ dense core must have spent a long time, much longer than the free-fall timescale, as a starless (i.e., pre-protostellar) core. The protostellar age of HH 34 MMS (HOPS 188) is unclear and may be in the order of $10^{5}$ years (Appendix). Therefore, the lifetime of some starless cores must be long enough to form millimeter-sized dust grains.

The evolution of dense core is governed by the physical process providing support against gravity. Magnetically supported cores may form stars in ambipolar diffusion timescales (Shu et al. 1987). By contrast, gravoturbulent star formation theory suggests that dense cores are transient objects supported by turbulence and collapse rapidly to form stars in free-fall timescales (Mac Low \& Klessen 2004; Klessen et al. 2005). The dense cores containing large dust grains found in this study seem to be counterexamples to the gravoturbulent theory. Detailed study of these cores may provide information on how they were supported during the pre-protostellar core stage.

Recent studies suggested that dust grains can play important roles in the structure and stability of dense cores (Whitworth \& Bate 2002; Seo \& Youdin 2016; Bate \& Lorén-Aguilar 2017). If the starless stage of dense cores can last long enough to produce large dust grains, there are interesting implications on the evolution of the cores. Such cores are likely to be in a quasistatic or marginally subcritical state, because substantially 
supercritical or subcritical cores may collapse or dissipate, respectively, in relatively short timescales. Large grains can occupy a significant fraction of the total dust mass, though they are small in numbers and invisible in extinction studies. The drag force of gas becomes ineffective, and the grains can be dynamically decoupled. At a density of $10^{5} \mathrm{~cm}^{-3}$ and temperature of $10 \mathrm{~K}$, grains larger than $\sim 160 \mu \mathrm{m}$ are decoupled from gas. Charged large grains are also decoupled from magnetic fields as the gyration period becomes very long. Because these grains are not supported by gas pressure, turbulence, or magnetic fields, they precipitate down and arrive at the center of the dense core in about $\sqrt{2}$ free-fall timescale. While completely decoupled grains oscillate or orbit around the center, moderately decoupled ones would settle in the highdensity region (Bate \& Lorén-Aguilar 2017). As a result, the concentration of large grains increases in the central region. The high dust concentration also enhances cooling of gas. Consequently, the density of the central region increases, and the core becomes unstable, which can trigger gravitational collapse. In this picture, the lifetime of starless dense cores can be affected or even limited by the production of large grains. The change of stability condition related with the inward migration of dust was studied by Whitworth \& Bate (2002), in the context of external radiation pressure. Hopkins (2014) discussed the collapse process of cores with large dust-to-gas ratios. Numerical simulations of large grains so far have investigated dynamics or growth of grains in already unstable and collapsing cores (Wong et al. 2016; Bate \& Lorén-Aguilar 2017). Simulations of large grains in quasistatic cores will be useful in understanding star formation activities in low- $\beta$ cores such as V380 Ori NE, HH 34 MMS, and HH 147 MMS. It is important to consider the precipitation, concentration, and growth of grains together, because these processes may affect each other. Observational searches for starless cores with low $\beta$ values will also be helpful in understanding the structure and evolution of the cores containing large grains.

\subsection{Chemical Signatures of Formation History}

The old age of a dense core can leave some chemical signatures owing to the depletion of molecules and grainsurface chemical reactions. Though a quantitative analysis of the chemical age is not easy, comparisons among dense cores can provide indications of relative age. Kang et al. (2015) found that the deuterium fractionation of the V380 Ori NE core $\left([\mathrm{HDCO}] /\left[\mathrm{H}_{2} \mathrm{CO}\right]=0.12\right)$ is higher than that of typical Class 0 protostellar cores $\left([\mathrm{HDCO}] /\left[\mathrm{H}_{2} \mathrm{CO}\right]=0.07\right)$, which suggests that this core has a history of high depletion of $\mathrm{CO}$ and $\mathrm{H}_{2} \mathrm{CO}$ (Bacmann et al. 2003).

The V380 Ori NE outflow shows the strongest $\mathrm{CH}_{3} \mathrm{OH}$ thermal emission among the 99 low-mass protostars in Orion surveyed by Kang et al. (2013). The $\mathrm{CH}_{3} \mathrm{OH}$ peak position (KLC 8) corresponds to the brightest $\mathrm{SiO}$ outflow peak r6 (Figure 1). This positional coincidence is consistent with the explanation that the $\mathrm{CH}_{3} \mathrm{OH}$ abundance is enhanced in protostellar outflows through desorption or sputtering from grain surfaces by hot gas in the shocked region (Bachiller et al. 1995; Kristensen et al. 2010). Interstellar $\mathrm{CH}_{3} \mathrm{OH}$ molecules are mainly formed on grain surfaces through hydrogenation of $\mathrm{CO}$ at low temperature (Fuchs et al. 2009). Cuppen et al. (2009) found that $\mathrm{CH}_{3} \mathrm{OH}$ molecules form efficiently in cold dense cores and dominate the ice layer of grain mantle with time. The strong $\mathrm{CH}_{3} \mathrm{OH}$ emission of V380 Ori NE agrees with the low dust emissivity index, in that this core may have spent a long time in the pre-protostellar core stage.

\section{Summary}

The star formation activities in the V380 Ori NE region were investigated by observing in the $\mathrm{SiO} v=0 J=1 \rightarrow 0$ line with VLA, in the $\mathrm{H}^{13} \mathrm{CO}^{+} J=1 \rightarrow 0, \mathrm{C}^{34} \mathrm{~S} J=2 \rightarrow 1$, and CO $J=1 \rightarrow 0$ lines with TRAO, and in the $\lambda=2.0,1.4,1.1$, and $0.89 \mathrm{~mm}$ continuum with CSO. The highly collimated $\mathrm{SiO}$ jet of V380 Ori NE was imaged with a resolution of 1."6, and the detailed structure of the jet was examined. The full extent of the V380 Ori NE bipolar outflow was measured using the CO map covering a $10^{\prime} \times 10^{\prime}$ region. The continuum map covers a $60^{\prime} \times 60^{\prime}$ region, and the dust continuum spectra of several dense cores were examined to investigate the dust properties. The main results are summarized as follows.

1. The $\mathrm{SiO}$ map shows a highly collimated bipolar jet, and emission peaks along the jet were identified. The $\mathrm{SiO}$ emission peaks show point-symmetric oscillation patterns in both the position angle and the velocity, when plotted as functions of distance from the driving source. The point symmetry suggests that the cause of the outflow variability is intrinsic to the driving source, and the most likely explanation is the precession of the jet axis. The length of the jet in the $\mathrm{SiO}$ image is longer than a full cycle of the jet precession.

2. The oscillation patterns of the precessing jet were fitted with a set of sinusoidal functions to derive the quantities describing the variability. From the best-fit functions, the wavelength of oscillation is $28^{\prime \prime}$, the oscillation amplitude of position angle is 3.5 , the amplitude of velocity oscillation is $2.0 \mathrm{~km} \mathrm{~s}^{-1}$, and the mean line-of-sight velocity is $8.2 \mathrm{~km} \mathrm{~s}^{-1}$ with respect to the systemic velocity. These variability parameters were converted to physical parameters describing the precessing jet, assuming that the flow elements are on the surface of a circular cone and have a uniform speed. The derived flow speed is $35 \mathrm{~km} \mathrm{~s}^{-1}$, and the precession cone has an inclination angle of $13^{\circ} .5$ and a half-opening angle of $3^{\circ} .4$.

3. The jet parameters provide useful information on the evolutionary status of the V380 Ori NE system. From the flow speed, inclination angle, and oscillation wavelength, the jet precession period is 1600 years. The full length of the outflow in the $\mathrm{CO}$ map corresponds to $\sim 3.9$ precession cycles, and the dynamical timescale of the V380 Ori NE outflow is $\sim 6300$ years. Because the inclination angle was estimated explicitly, the outflow timescale may be a good estimation of the protostellar age. Considerations of the age and the flow speed suggest that the mass of the protostar may be $\sim 0.02 M_{\odot}$. The outflow timescale and the mass estimate confirm that the protostar is extremely young, as previously inferred from the SED (Stutz et al. 2013).

4. The jet precession suggests that V380 Ori NE may be a protostellar binary system with a misalignment between the disk and the orbital plane. Two modes of tidal perturbation can be considered. If the precession of disk is responsible for the observed precession of the $\mathrm{SiO}$ jet, the orbital period and separation of the binary system may be $\sim 80$ years and $\sim 6$ au, respectively. If the wobbling of disk is the responsible mechanism, the 
corresponding values may be $\sim 3200$ years and $\sim 70$ au. These estimates of binary separation suggest that the presumed V380 Ori NE binary system may have been formed through the disk instability process.

5. The MUSIC continuum maps show several sources in the L1641 region. Eight sources were identified using the 1.4 and $1.1 \mathrm{~mm}$ continuum maps. Their spectral slopes suggest that the detected flux densities come almost entirely from the thermal dust emission. Three dense cores, V380 Ori NE, HH 34 MMS, and HH 147 MMS, show particularly shallow SED slopes.

6. The SED of each dense core was fitted with a modified blackbody function, assuming a power-law emissivity $\left(\kappa_{\nu} \propto \nu^{\beta}\right)$. In addition to the MUSIC data, the $850 \mu \mathrm{m}$ flux densities of Nutter \& Ward-Thompson (2007) were used. The single-component dust emission models fit the data reasonably well. The emissivity index $\beta$ and mass of each core were derived assuming a dust temperature of $20 \mathrm{~K}$. While the $\beta$ values of the majority of dense cores are consistent with those of other dense cores in the literature, V380 Ori NE, HH 34 MMS, and HH 147 MMS have unusually low values: $\beta=0.3,0.7$, and 0.7 , respectively. Considering the relatively large uncertainties, these low $\beta$ values are tentative. Because such low values $(\beta<1)$ imply that the maximum size of dust grains may be at least a few times the wavelength observed (Draine 2006), these three cores may contain a substantial amount of millimeter-sized dust grains.

7. The detected dense cores show a positive correlation between the dust emissivity index and molecular line width. The strength of turbulence may be related with the dust size distribution by affecting the shattering and coagulation processes of dust grains. The emissivity index has no strong correlation with the bolometric luminosity or temperature of the protostars.

8. Growing dust grains to a large size $(>100 \mu \mathrm{m})$ takes millions of years, much longer than the free-fall timescale (Ormel et al. 2009). By contrast, the age of the protostars in V380 Ori NE and HH 34 MMS are shorter. In particular, the protostellar age of V380 Ori NE, inferred from the outflow timescale, is only about 6300 years, which is much shorter than the free-fall timescale. The stark difference between the grain growth timescale and the protostellar age suggests that these cores should already have produced large dust grains during their starless core or pre-protostellar core stage of evolution. Therefore, the lifetime of some starless dense cores may be much longer than the free-fall timescale. The large dust grains may be an interesting component of dense cores because they are dynamically decoupled from gas (Bate \& Lorén-Aguilar 2017). Because they can destabilize starless cores by affecting the density structure and cooling process, they may play an important role in the evolution of dense cores.

We thank the TRAO and the CSO staffs for their support. NRAO is a facility of the National Science Foundation operated under cooperative agreement by Associated Universities, Inc. M. Kang was supported by Basic Science Research Program through the National Research Foundation of Korea funded by the Ministry of Science and ICT (grant No. NRF-2015R1C1A 1A01052160). J.-E. Lee was supported by the Basic Science
Research Program through the National Research Foundation of Korea (grant No. NRF-2015R1A2A2A01004769) and the Korea Astronomy and Space Science Institute under the R\&D program (Project No. 2015-1-320-18) supervised by the Ministry of Science and ICT.

\section{Appendix Individual Dense Cores}

\section{A.1. L1641N MMS}

The brightest source in the MUSIC maps is referred to as L1641N MMS in this paper. It is a complex of several subcores and protostars. Stanke \& Williams (2007) imaged the core with a $\sim 1$ ". 4 resolution and identified sub-cores MM1-4. Furlan et al. (2016) identified three protostars in this core. The most deeply embedded one, HOPS 182, is a Class 0 YSO with $L_{\text {bol }}=71.1 L_{\odot}$, located at the sub-core MM1. The core shows relatively strong emission in the $\mathrm{CH}_{3} \mathrm{OH}$ and $\mathrm{CH}_{3} \mathrm{CN}$ lines (Stanke \& Williams 2007; Kang et al. 2013). Stanke \& Williams (2007) suggested that there is a hot corino around MM1. There are at least four bipolar CO outflows (Stanke \& Williams 2007; Nakamura et al. 2012) and an active $\mathrm{H}_{2} \mathrm{O}$ maser source (Kang et al. 2013).

L1641N MMS is the most extended source in the MUSIC maps. It is elongated in the northeast-southwest direction, with a position angle of $\sim 43^{\circ}$ and a deconvolved size of $\sim 44^{\prime \prime}(0.09$ pc). The emissivity index $\beta=1.4$ is near the median value among the detected cores. There is a secondary peak at $\sim 55^{\prime \prime}$ northeast of the main peak (Figure 10(c)). Though there is no known protostar in the secondary peak, the $\mathrm{H}_{2} \mathrm{O}$ maser reported by Xiang \& Turner (1995) is probably associated with it.

\section{A.2. Strom 11}

Strom 11 is a group of embedded near-infrared objects associated with IRAS 05339-0626 (Strom et al. 1989; Chen et al. 1993). There is a blueshifted CO outflow in this region (Stanke \& Williams 2007; Nakamura et al. 2012). Furlan et al. (2016) identified five protostars in this core, all with relatively low (less than $2 L_{\odot}$ ) luminosities. Two of them (HOPS 173 and 380) are Class 0 YSOs.

The Strom 11 dense core has the steepest spectral slope and the highest emissivity index $(\beta=2.2)$ among the detected cores. Even if a higher dust temperature is used for the calculation, the emissivity index remains high. Assuming $T_{d}=40 \mathrm{~K}$, for example, the SED fit gives $\beta=2.1$. This core does not seem to contain large dust grains.

\section{A.3. TUKH 74}

TUKH 74 is the only starless core among the detected cores. The emissivity index $\beta=1.4$ is near the median value. Considering that it does not have an internal heating source, the dust temperature could be relatively low. However, Ohashi et al. (2014) derived a kinetic temperature of $17 \mathrm{~K}$ from the $\mathrm{NH}_{3}$ data of Wilson et al. (1999). Therefore, the assumed dust temperature of $20 \mathrm{~K}$ is reasonable. In the grain model of Ricci et al. (2010), $\beta=1.4$ implies a maximum grain size of $\sim 1 \mathrm{~mm}$. Considering the uncertainty, it is unclear whether this starless core contains large dust grains. 


\section{A.4. $H H 34 M M S$}

The core associated with the $\mathrm{HH} 34$ jet is referred to as $\mathrm{HH}$ 34 MMS in this paper. There are two sub-cores that are not clearly separated by the MUSIC beams (see Figure 14 of Johnstone \& Bally 2006). The main core contains the driving source of $\mathrm{HH} \mathrm{34}$, and the secondary core contains IRS 5 . Furlan et al. (2016) identified three Class I protostars in this region. HOPS 188 has $L_{\mathrm{bol}}=18.8 L_{\odot}$ and $T_{\mathrm{bol}}=103 \mathrm{~K}$ and corresponds to the driving source of the HH 34 jet. Because the bolometric temperature is not much higher than the Class $0 / \mathrm{I}$ boundary $(70 \mathrm{~K})$, the age of this protostar may be somewhat older than the typical lifetime of the Class 0 phase $\left(\sim 10^{5}\right.$ years; Evans et al. 2009). The prominent HH 34 jet has been studied extensively (Reipurth et al. 1986, 2002; Rodríguez \& Reipurth 1996; Nisini et al. 2016). The jet extends to a parsec-scale outflow that has a dynamic age of $\sim 10,000$ years (Bally \& Devine 1994; Devine et al. 1997). Considering both the YSO classification and outflow timescale, the protostellar age of HOPS 188 may be on the order of $10^{5}$ years. HOPS 189 corresponds to IRS 5 and has $L_{\mathrm{bol}}=1.3 L_{\odot}$. HOPS 190 corresponds to Object 22 of Reipurth et al. $(1986,2002)$ and has $L_{\mathrm{bol}}=0.4 L_{\odot}$.

Most of the emission detected by MUSIC comes from the main core. The low emissivity index $\beta=0.7$ suggests that this core contains millimeter-sized dust grains.

\section{A.5. V380 Ori NE}

V380 Ori NE is a relatively isolated core. Furlan et al. (2016) identified a protostar in this core: HOPS 169 is a Class 0 YSO with $L_{\mathrm{bol}}=3.9 L_{\odot}$ and $T_{\mathrm{bol}}=33 \mathrm{~K}$. The star formation activities of V380 Ori NE are described in the main text.

V380 Ori NE has the lowest dust emissivity index, $\beta=0.3$, among the detected cores, which suggests that it contains millimeter-sized dust grains. Davis et al. (2000) suggested that the submillimeter emission from the extended part of the core probably comes from dust heated by the outflow. If the dust temperature is higher, however, it would make $\beta$ even lower. Assuming $T_{d}=30 \mathrm{~K}$, for example, the SED fit gives $\beta=0.2$.

\section{A.6. $\mathrm{HH} 147 \mathrm{MMS}$}

HH 147 MMS is the dense core containing $\mathrm{N}^{3}$ SK 50, the driving source of the HH 147 outflow (Nakajima et al. 1986; Eislöffel et al. 1994; Chini et al. 1997, 2001; Choi \& Zhou 1997). The nature of this YSO is unclear. It was known to be a rare type of T Tauri star exhibiting a $\mathrm{P}$ Cygni profile in $\mathrm{H} \alpha$ (Strom et al. 1989; Corcoran \& Ray 1995). By contrast, Chini et al. (2001) noted that the bolometric-to-submillimeter luminosity ratio of the core indicates a Class 0 YSO. Furlan et al. (2016) classified its counterpart, HOPS 166, as a flatspectrum YSO with $L_{\mathrm{bol}}=15.5 L_{\odot}$ and $T_{\mathrm{bol}}=457 \mathrm{~K}$. MoroMartín et al. (1999) detected a CO outflow.

The emissivity index is low $(\beta=0.7)$, but the uncertainty is relatively large. This core probably contains millimeter-sized dust grains.

\section{A.7. $\mathrm{HH}$ 1-2 MMS 2}

The HH 1-2 MMS 2 core in the MUSIC maps includes MMS 2 and MMS 3 of Chini et al. (1997, 2001). There are two compact sources, VLA 3 and VLA 6, detected in centimeter continuum (Rodríguez et al. 1990, 2000). VLA 3 corresponds to HOPS 168, a Class 0 YSO with $L_{\mathrm{bol}}=48.1 L_{\odot}$ (Furlan et al. 2016). This protostar displays $\mathrm{H}_{2} \mathrm{O}$ maser activities (Lo et al. 1975; Kang et al. 2013). There is a powerful CO outflow in this region (Chernin \& Masson 1995; Choi \& Zhou 1997; MoroMartín et al. 1999). VLA 6 is a time-variable source and probably a magnetically active YSO (Rodríguez et al. 2000). Furlan et al. (2016) identified yet another protostar located between MMS 1 and MMS 2: HOPS 167 corresponds to source 21 of Kwon et al. (2010) and has $L_{\text {bol }}=0.2 L_{\odot}$.

HH 1-2 MMS 2 has a relatively high dust emissivity index $(\beta=1.9)$. This core does not seem to contain large dust grains.

\section{A.8. HH 1-2 MMS 1}

HH 1-2 MMS 1 is the dense core containing the driving source of the archetypal Herbig-Haro objects HH 1 and HH 2 (Chini et al. 1997, 2001). The optical jet, HH objects, and their environment have been studied extensively (Strom et al. 1989; Reipurth et al. 1993; Eislöffel et al. 1994; Corcoran \& Ray 1995; Kwon et al. 2010). There are three compact sources, VLA $1 / 2 / 4$, detected in centimeter continuum (Rodríguez et al. 2000). VLA 1 corresponds to HOPS 203, a Class 0 YSO with $L_{\mathrm{bol}}=20.4 L_{\odot}$ (Furlan et al. 2016). It is the driving source of the HH 1-2 outflow. The $\mathrm{CO}$ outflow associated with the HH 1-2 jet is relatively weak (Chernin \& Masson 1995; Choi \& Zhou 1997; Moro-Martín et al. 1999). The $\mathrm{H}_{2} \mathrm{O}$ maser activities reported by Kang et al. (2013) is probably associated with a VLA 1 or HH 1 jet. VLA 2 probably drives the HH 144 outflow, but its nature is unclear (Reipurth et al. 1993; Eislöffel et al. 1994). VLA 4 corresponds to HOPS 165, a Class I YSO with $L_{\mathrm{bol}}=3.4 L_{\odot}$ (Furlan et al. 2016). It is probably the driving source of the HH 146 outflow (Reipurth et al. 1993).

The HH 1-2 MMS 1 dense core has a relatively high dust emissivity index $(\beta=1.6)$. The MMS 1 core does not seem to contain large dust grains.

The three cores (HH 1-2 MMS 1 and 2, and HH 147 MMS) are spatially close to each other, and their star-forming activities may be considered as a single cluster-forming event. Kwon et al. (2010) found that the magnetic fields in this region are regular and straight, but the outflow orientations are essentially random. This contrast between magnetic fields and outflows suggests that the star formation in this region is mainly driven by turbulence. As predicted by the gravoturbulent star formation theory, the ages of the HH 1-2 MMS 1 and 2 cores are probably too short to form large dust grains.

\section{ORCID iDs}

Miju Kang (1) https://orcid.org/0000-0002-5016-050X Jeong-Eun Lee (으 https://orcid.org/0000-0003-3119-2087 Ken'ichi Tatematsu (ํ) https://orcid.org/0000-0002-8149-8546 Sung-Ju Kang (1) https://orcid.org/0000-0002-5004-7216 Jack Sayers (1) https://orcid.org/0000-0002-8213-3784 Jungyeon Cho (i) https://orcid.org/0000-0003-1725-4376 Jungmi Kwon (1) https://orcid.org/0000-0003-2815-7774 Geumsook Park @it https://orcid.org/0000-0001-8467-3736

\section{References}

André, P., Men'shchikov, A., Bontemps, S., et al. 2010, A\&A, 518, L102 Bachiller, R., Liechti, S., Walmsley, C. M., \& Colomer, F. 1995, A\&A, 295, L51

Bacmann, A., Lefloch, B., Ceccarelli, C., et al. 2003, ApJL, 585, L55 Bally, J., \& Devine, D. 1994, ApJL, 428, L65

Bate, M. R., Bonnell, I. A., Clarke, C. J., et al. 2000, MNRAS, 317, 773 
Bate, M. R., Lodato, G., \& Pringle, J. E. 2010, MNRAS, 401, 1505

Bate, M. R., \& Lorén-Aguilar, P. 2017, MNRAS, 465, 1089

Baudry, A., Lucas, R., \& Guilloteau, S. 1995, A\&A, 293, 594

Cabrit, S., Codella, C., Gueth, F., et al. 2007, A\&A, 468, L29

Chen, H., Tokunaga, A. T., Strom, K. M., \& Hodapp, K.-W. 1993, ApJ, 407, 639

Chen, M. C.-Y., Di Francesco, J., Johnstone, D., et al. 2016, ApJ, 826, 95

Chernin, L. M., \& Masson, C. R. 1995, ApJ, 443, 181

Chini, R., Reipurth, B., Sievers, A., et al. 1997, A\&A, 325, 542

Chini, R., Ward-Thompson, D., Kirk, J. M., et al. 2001, A\&A, 369, 155

Choi, M. 2005, ApJ, 630, 976

Choi, M., Kang, M., \& Tatematsu, K. 2011a, ApJL, 728, L34

Choi, M., Kang, M., Tatematsu, K., Lee, J.-E., \& Park, G. 2011b, PASJ, 63, 1281

Choi, M., \& Zhou, S. 1997, ApJ, 477, 754

Chokshi, A., Tielens, A. G. G. M., \& Hollenbach, D. 1993, ApJ, 407, 806

Codella, C., Cabrit, S., Gueth, F., et al. 2007, A\&A, 462, L53

Corcoran, D., \& Ray, T. P. 1995, A\&A, 301, 729

Coupeaud, A., Demyk, K., Meny, C., et al. 2011, A\&A, 535, A124

Cuppen, H. M., van Dishoeck, E. F., Herbst, E., \& Tielens, A. G. G. M. 2009, A\&A, 508, 275

Davis, C. J., Dent, W. R. F., Matthews, H. E., Coulson, I. M., \& McCaughrean, M. J. 2000, MNRAS, 318, 952

Davis, C. J., Froebrich, D., Stanke, T., et al. 2009, A\&A, 496, 153

Dent, W. R. F., Matthews, H. E., \& Ward-Thompson, D. 1998, MNRAS, 301, 1049

Devine, D., Bally, J., Reipurth, B., \& Heathcote, S. 1997, AJ, 114, 2095

Downes, T. P., \& Cabrit, S. 2007, A\&A, 471, 873

Draine, B. T. 2006, ApJ, 636, 1114

Dutrey, A., Guilloteau, S., \& Bachiller, R. 1997, A\&A, 325, 758

Eislöffel, J., \& Mundt, R. 1997, AJ, 114, 280

Eislöffel, J., Mundt, R., \& Böhm, K.-H. 1994, AJ, 108, 1042

Erickson, N. R., Grosslein, R. M., Erickson, R. B., \& Weinreb, S. 1999 ITMTT, 47, 2212

Evans, N. J., II, Dunham, M. M., Jørgensen, J. K., et al. 2009, ApJS, 181, 321

Fendt, C., \& Zinnecker, H. 1998, A\&A, 334, 750

Froebrich, D. 2005, ApJS, 156, 169

Fuchs, G. W., Cuppen, H. M., Ioppolo, S., et al. 2009, A\&A, 505, 629

Furlan, E., Fischer, W. J., Ali, B., et al. 2016, ApJS, 224, 5

Gibb, A. G., Richer, J. S., Chandler, C. J., \& Davis, C. J. 2004, ApJ, 603, 198

Golwala, S. R., Bockstiegel, C., Brugger, S., et al. 2012, Proc. SPIE, 8452, 5

Henning, T., Michel, B., \& Stognienko, R. 1995, P\&SS, 43, 1333

Hildebrand, R. H. 1983, QJRAS, 24, 267

Hirano, N., Liu, S.-Y., Shang, H., et al. 2006, ApJL, 636, L141

Hirashita, H., \& Yan, H. 2009, MNRAS, 394, 1061

Hodapp, K. W., Bally, J., Eislöffel, J., \& Davis, C. J. 2005, AJ, 129, 1580

Hogerheijde, M. R., \& Sandell, G. 2000, ApJ, 534, 880

Hopkins, P. F. 2014, ApJ, 797, 59

Hopkins, P. F., \& Lee, H. 2016, MNRAS, 456, 4174

Hsu, W.-H., Hartmann, L., Allen, L., et al. 2012, ApJ, 752, 59

Hsu, W.-H., Hartmann, L., Allen, L., et al. 2013, ApJ, 764, 114

Jackson, J. M., Rathborne, J. M., Shah, R. Y., et al. 2006, ApJS, 163, 145

Johnstone, D., \& Bally, J. 2006, ApJ, 653, 383

Kang, M., Choi, M., Stutz, A. M., \& Tatematsu, K. 2015, ApJ, 814, 31

Kang, M., Lee, J.-E., Choi, M., et al. 2013, ApJS, 209, 25

Kim, M. K., Hirota, T., Honma, M., et al. 2008, PASJ, 60, 991

Klessen, R. S., Ballesteros-Paredes, J., Vázquez-Semadeni, E., \& Durán-Rojas, C. 2005, ApJ, 620, 786

Kratter, K., \& Lodato, G. 2016, ARA\&A, 54, 271

Kristensen, L. E., van Dishoeck, E. F., van Kempen, T. A., et al. 2010, A\&A, 516, A57

Kwon, J., Choi, M., Pak, S., et al. 2010, ApJ, 708, 758

Kwon, W., Looney, L. W., Mundy, L. G., Chiang, H.-F., \& Kemball, A. J. 2009, ApJ, 696, 841

Lai, D. 2003, ApJL, 591, L119

Lee, C.-F., Hirano, N., Zhang, Q., et al. 2015, ApJ, 805, 186
Lee, J.-E., Lee, S., Dunham, M. M., et al. 2017, NatAs, 1, 0172 Levreault, R. M. 1988, ApJS, 67, 283

Lo, K. Y., Burke, B. F., \& Haschick, A. D. 1975, ApJ, 202, 81 López-Sepulcre, A., Watanabe, Y., Sakai, N., et al. 2016, ApJ, 822, 85 Mac Low, M.-M., \& Klessen, R. S. 2004, RvMP, 76, 125 Machida, M. N., Inutsuka, S.-I., \& Matsumoto, T. 2008, ApJ, 676, 1088 Masunaga, H., \& Inutsuka, S.-I. 2000, ApJ, 531, 350

Miotello, A., Testi, L., Lodato, G., et al. 2014, A\&A, 567, A32

Morgan, J. A., Schloerb, F. P., Snell, R. L., \& Bally, J. 1991, ApJ, 376, 618

Morgan, J. A., Snell, R. L., \& Strom, K. M. 1990, ApJ, 362, 274

Moro-Martín, A., Cernicharo, J., Noriega-Crespo, A., \& Martín-Pintado, J. 1999, ApJL, 520, L111

Myers, P. C., Adams, F. C., Chen, H., \& Schaff, E. 1998, ApJ, 492, 703

Nakajima, T., Nagata, T., Nishida, M., Sato, S., \& Kawara, K. 1986, MNRAS, 221,483

Nakamura, F., Miura, T., Kitamura, Y., et al. 2012, ApJ, 746, 25

Nisini, B., Giannini, T., Antoniucci, S., et al. 2016, A\&A, 595, A76

Nutter, D., \& Ward-Thompson, D. 2007, MNRAS, 374, 1413

Offner, S. S. R., Kratter, K. M., Matzner, C. D., Krumholz, M. R., \& Klein, R. I. 2010, ApJ, 725, 1485

Ohashi, S., Tatematsu, K., Choi, M., et al. 2014, PASJ, 66, 119

Ormel, C. W., Paszun, D., Dominik, C., \& Tielens, A. G. G. M. 2009, A\&A, 502,845

Ossenkopf, V., \& Henning, T. 1994, A\&A, 291, 943

Planck Collaboration, Ade, P. A. R., Aghanim, N., et al. 2014, A\&A, 564, A45

Reipurth, B., Bally, J., Graham, J. A., Lane, A. P., \& Zealey, W. J. 1986, A\&A, 164,51

Reipurth, B., Heathcote, S., Morse, J., Hartigan, P., \& Bally, J. 2002, AJ, 123, 362

Reipurth, B., Heathcote, S., Roth, M., Noriega-Crespo, A., \& Raga, A. C. 1993, ApJL, 408, L49

Ricci, L., Testi, L., Natta, A., et al. 2010, A\&A, 512, A15

Rodríguez, L. F., Delgado-Arellano, V. G., Gómez, Y., et al. 2000, AJ, 119,882

Rodríguez, L. F., Ho, P. T. P., Torrelles, J. M., Curiel, S., \& Cantó, J. 1990, ApJ, 352, 645

Rodríguez, L. F., \& Reipurth, B. 1996, RMxAA, 32, 27

Sadavoy, S. I., Di Francesco, J., Johnstone, D., et al. 2013, ApJ, 767, 126

Sadavoy, S. I., Stutz, A. M., Schnee, S., et al. 2016, A\&A, 588, A30

Saigo, K., Tomisaka, K., \& Matsumoto, T. 2008, ApJ, 674, 997

Sayers, J., Bockstiegel, C., Brugger, S., et al. 2014, Proc. SPIE, 9153, 4

Sayers, J., Zemcov, M., Glenn, J., et al. 2016, ApJ, 820, 101

Schnee, S., Mason, B., Di Francesco, J., et al. 2014, MNRAS, 444, 2303

Seo, Y. M., \& Youdin, A. N. 2016, MNRAS, 461, 1088

Sheikhnezami, S., \& Fendt, C. 2015, ApJ, 814, 113

Shirley, Y. L., Evans, N. J., II, \& Rawlings, J. M. C. 2002, ApJ, 575, 337

Shu, F. H., Adams, F. C., \& Lizano, S. 1987, ARA\&A, 25, 23

Spalding, C., Batygin, K., \& Adams, F. C. 2014, ApJL, 797, L29

Stanke, T., McCaughrean, M. J., \& Zinnecker, H. 2002, A\&A, 392, 239

Stanke, T., \& Williams, J. P. 2007, AJ, 133, 1307

Strom, K. M., Newton, G., Strom, S. E., et al. 1989, ApJS, 71, 183

Strom, K. M., Strom, S. E., \& Merrill, K. M. 1993, ApJ, 412, 233

Stutz, A. M., Tobin, J. J., Stanke, T., et al. 2013, ApJ, 767, 36

Tatematsu, K., Umemoto, T., Kameya, O., et al. 1993, ApJ, 404, 643

Terquem, C., Eislöffel, J., Papaloizou, J. C. B., \& Nelson, R. P. 1999, ApJL, 512, L131

Testi, L., Birnstiel, T., Ricci, L., et al. 2014, in Protostars and Planets VI ed. H. Beuther et al. (Tucson, AZ: Univ. Arizona Press), 339

Tobin, J. J., Looney, L. W., Li, Z.-Y., et al. 2016, ApJ, 818, 73

Tsukamoto, Y., \& Machida, M. N. 2013, MNRAS, 428, 1321

Visser, A. E., Richer, J. S., Chandler, C. J., \& Padman, R. 1998, MNRAS, 301,585

Whitworth, A. P., \& Bate, M. R. 2002, MNRAS, 333, 679

Wilson, T. L., Mauersberger, R., Gensheimer, P. D., Muders, D., \& Bieging, J. H. 1999, ApJ, 525, 343

Wong, Y. H. V., Hirashita, H., \& Li, Z.-Y. 2016, PASJ, 68, 67

Xiang, D., \& Turner, B. E. 1995, ApJS, 99, 121 